\title{
Contracting with Private Rewards
}

\author{
René Kirkegaard \\ University of Guelph \\ rkirkega@uoguelph.ca \\ Department of Economics and Finance \\ University of Guelph \\ Discussion Paper 2015-04
}




\title{
Contracting with Private Rewards*
}

\author{
René Kirkegaard \\ Department of Economics and Finance \\ University of Guelph \\ rkirkega@uoguelph.ca.
}

May 2015

\begin{abstract}
I extend the canonical moral hazard model to allow the agent to face endogenous and non-contractible uncertainty. The agent works for the principal and simultaneously pursues private rewards. I establish conditions under which the first-order approach remains valid. The model adds to the literature on intrinsic versus extrinsic motivation. Specifically, to induce higher effort at work the contract may offer higher rewards but flatter incentives. The contract change makes the agent reevaluate his "worklife balance". Larger employment rewards lessens the incentive to pursue private rewards. The greater reliance on labor income then necessitates weaker explicit incentives to induce high effort.
\end{abstract}

JEL Classification Numbers: D82, D86

Keywords: First-Order Approach, Intrinsic Motivation, Moral Hazard, Multi-tasking, Principal-Agent Models, Private Rewards.

*I thank the Canada Research Chairs programme and SSHRC for funding this research. I am grateful for comments and suggestions from seminar audiences at Queen's University, the University of Guelph, and the Canadian Economic Theory Conference. 


\section{Introduction}

The principal-agent model has been tremendously influential in economics. However, the canonical model essentially assumes that the principal-agent relationship takes place in a perfect vacuum - there are no (non-contractible) outside random disturbances. For instance, the only payoff-relevant risk the agent faces is due to the uncertainty embodied in the incentive scheme offered by the principal.

In reality, however, it is easier to think of examples in which the agent faces some non-contractible outside uncertainty than examples in which this is not the case. Indeed, such uncertainty is often endogenous. That is, the agent pursues a host of potentially rewarding activities that are not directly observable (nor necessarily directly relevant) to the principal. Even seemingly mundane activities may in reality entail significant rewards. For instance, when the busy young professional tolerates dinner with her parents, she may hope to join the " 27 percent of those purchasing a home for the first time [who] received a cash gift from relatives or friends to come up with a down payment." ${ }^{1}$ When her older brother moves his family closer to their parents at the cost of a longer commute, he may be motivated by the fact that "by the time the average youngster reaches school age, they will have been babysat by their grandparents for more than 5,610 hours." 2 The "rewards" the parents bestow upon their children are most likely not observable to employers; they are non-contractible.

There are a plethora of other examples in which the agent directly receives a reward from a third party. Although the waiter has an employment contract with the restaurant owner, a significant part of her income often comes in the form of tips from the diner, despite the fact that there is no explicit contract between the two (nor is there an explicit contract between the parents and offspring in the previous paragraph). In other cases, the agent is in a formal contractual relationship with more than one principal, a situation known as common agency. Thus, developing an understanding of contracting with private rewards is a necessary

\footnotetext{
${ }^{1}$ The data is for the U.S, in 2013. See www.bloomberg.com/news/2014-09-19/mom-anddad-banks-step-up-aid-to-first-time-home-buyers.html

${ }^{2}$ The data is for the U.K. The estimated monetary value of this amount of child care is $£ 21,654.60$. See http://www.dailymail.co.uk/femail/article-2263843/The-21-000-grandmaGrandparents-babysitting-duties-reduce-cost-childcare-whopping-4-300-EVERY-YEAR.html
} 
first step towards analyzing common agency environments in which principals do not have access to the same information.

Examples involving potentially large non-monetary rewards include the agent's health status as impacted by endogenous life-style choices, his social status in his peer group, the quality of his match on the marriage market as affected by his search intensity, and so on. The satisfaction from mastering a second language, or any other hobby, is another example. Even the agent's "job-satisfaction" may be endogenous, influenced by the enthusiasm with which he interacts with colleagues. Typically, uncertainty over the private rewards is fully resolved only after the contract is signed. Hence, as private rewards and labor income interact in his utility function, the agent is uncertain even of his own marginal utility of labor income at the time of contracting.

The aim of this paper is to analyze the consequences of endogenous "private rewards" on optimal contracting. The standard principal-agent model is amended to allow the agent to work on two tasks. The first "task" captures the effort the agent expends working on behalf of the principal. This task produces a contractible signal, as in the standard model. The second task describes the effort devoted to pursuing private rewards (which the principal may or may not directly care about). This task does not produce a contractible signal. Thus, the agent is multi-tasking, but the principal observes only the outcome of one task before compensating the agent. ${ }^{3}$

The formal contract offered by the principal combines with the promise of external rewards to form a mixed stew of incentives that ultimately determines how hard the agent works on both tasks. Now, in a recent survey of behavioral contract theory, Köszegi (2014) singles out "the literature on the interaction between extrinsic and intrinsic motivation [as] one of the most exciting and productive in behavioral contract theory." In this literature, intrinsic motivation refers to nonmonetary reasons why the agent would work hard on behalf of the principal. The call for more research is accompanied by the observation that "unlike extrinsic motivation, intrinsic motivation is a complex multifaceted phenomenon that is poorly understood." In the current paper, it is also the case that the contract

\footnotetext{
${ }^{3}$ In the following, the term "action" refers to the pair of efforts devoted to the two different tasks. Conversely, a "task" describes one particular dimension of the action.
} 
does not capture all that is payoff-relevant to the agent. Thus, one "facet" of what looks like "intrinsic motivation" to the outsider may be that the agent has to evaluate how rewards on the job interact with private rewards.

Indeed, the model can be interpreted as endogenizing the agent's pursuit of "work-life balance." The standard single-task model essentially focuses on the work dimension. In that model, the cost function may capture foregone leisure. However, given the separability that is usually assumed, the value of "leisure" is determined solely by effort at work but is otherwise independent of the contract. The current model, however, allows the agent to simultaneously invest in both dimensions - "work" and "life" - while recognizing that the contract may influence both decisions. Stated differently, in the standard model no consideration is given to how exactly the agent spends his time when he is not working; leisure is no more than a black-box residual. Here, in contrast, the agent can decide how intensely or actively he utilizes his leisure time. If the agent is paid poorly at work - rewards from labor are low - he may decide to seek rewards elsewhere, by e.g. investing more heavily in a hobby. The implied multi-tasking turns out to alter some key predictions of standard contract theory.

The dominant method for analyzing moral hazard is the first-order approach (FOA). The FOA has been justified in a class of multi-tasking problems only very recently; see Kirkegaard (2014). ${ }^{4}$ In this paper, I build on this work to extend the FOA to handle private rewards. Although rewards are assumed to be stochastically independent, the model allows for interdependencies in two ways. First, effort costs may be non-separable in the two tasks. Second, rewards from the two different sources may be substitutes in the agent's utility function.

Thus, the first contribution of the paper is to present a tractable model of contracting in the presence of private rewards. The second contribution - justifying the FOA - is methodological in nature. That is, I provide a solution technique that can be used in future research on contracting with private rewards. Third,

\footnotetext{
${ }^{4}$ Holmström and Milgrom (1991) present a fairly specialized multi-task model in which the FOA is valid. Certain forms of private rewards can be accommodated. Likewise, Ábrahám et al (2011) justify the FOA in a model with hidden savings. The agent privately earns a return on his savings, but this is both deterministic and monetary. Detailed discussions of these papers are in Section 6.1 and Section 3, respectively. Ligon and Thistle (2013) introduce exogenous background risk into the standard model. In their setting, the agent's action is one-dimensional and can take only one of two values, thereby obviating the need for the FOA.
} 
the model is applied to provide a new perspective on intrinsic versus extrinsic motivation. Specifically, contracts that may seem to have "flatter" incentives yielding a smaller return to a marginal increase in on-the-job effort - may induce the agent to work harder on the job. Although this finding is at odds with the conventional wisdom, Kőszegi (2014) reviews several "behavioral economics" models in which results in this vein are obtained. Here, I identify a new mechanism, centered on considerations of "work-life balance", which is responsible for the result. Additionally, the model identifies a new source of economic rents for the agent. Thus, unlike in the standard model, the agent may earn more than his reservation utility. It is worth emphasizing that a popular competing model of multi-tasking is not rich enough to produce these predictions (see below).

To illustrate the last two points it is useful to further specialize the model. In the multiplicative model, the agent's expected utility takes the form $M\left(a_{1} \mid \omega\right) N\left(a_{2}\right)-$ $c\left(a_{1}, a_{2}\right)$, where $\omega$ denotes the contract, $a_{1}$ effort on the job, and $a_{2}$ effort in pursuit of private rewards. Here, $c$ is a cost function, while $M\left(a_{1} \mid \omega\right)$ describes expected utility from labor income. In the standard model, $a_{2}$ is essentially fixed and $N>0$ is an exogenous constant, but here it captures the expected impact of private rewards. The example in Section 2 can be shown to have this form. The multiplicative model applies if e.g. the private reward is monetary and the agent's preferences exhibits constant absolute risk aversion over total income.

It is not hard to see that the contract is determined entirely by the incentive compatibility constraints. The participation constraint is redundant on the set of implementable actions. Indeed, when the principal takes an interest in both tasks (but typically not otherwise), it may be optimal to implement an action for which the participation constraint is slack. Note that the agent's rents are not due to the outside rewards per se, but rather to the fact that they are private. After all, if the outside rewards are contractible, the principal can effectively appropriate their monetary value by making the agent's pay contingent on both signals. ${ }^{5}$

The comparative statics of the multiplicative model are rather tractable as

\footnotetext{
${ }^{5}$ There may be other reasons why the agent earns more than his reservation utility. This may occur if the agent is protected by limited liability. Moreover, Laffont and Martimort (2002, Section 5.3) explains how the agent may earn rents when his utility function is non-separable in income and effort. In this case the participation constraint is not redundant - it is just not optimal to make it binding. Here, I follow most of the literature by assuming separability.
} 
they rely primarily on incentive compatibility. Let $\omega^{\prime}$ and $\omega^{\prime \prime}$ denote two different contracts that induce the agent to deliver the same $a_{1}$ effort at work. It turns out that $M\left(a_{1} \mid \omega^{\prime \prime}\right)>M\left(a_{1} \mid \omega^{\prime}\right)$ if and only if $M^{\prime}\left(a_{1} \mid \omega^{\prime \prime}\right)<M^{\prime}\left(a_{1} \mid \omega^{\prime}\right)$, where $M^{\prime}\left(a_{1} \mid \omega\right)$ denotes the derivative of $M$ with respect to $a_{1}$. To an outsider who fails to recognize that $N$ is endogenous, it would thus seem that $\omega^{\prime \prime}$ delivers higher "base utility" than $\omega^{\prime}$ but weaker explicit or extrinsic incentives. Nevertheless, the agent works just as hard with the latter contract. Thus, the outsider might suspect that one of the behavioral models reviewed by Köszegi (2014) are at play.

For instance, Englmaier and Leider (2012) note that if the agent has reciprocal preferences, the principal can "generate intrinsic motivation" by giving the agent higher base utility. The agent reciprocates by maintaining high effort even if explicit incentives are weakened. In the simplest version of Bénabou and Tirole's (2003) model, the agent derives utility (a source of intrinsic motivation) if he performs well on the job. However, the agent only has an imperfect signal about the cost of effort. If the principal knows that effort is very costly, he may be worried that the agent has received a bad signal. Consequently, he is more likely to offer steeper explicit incentives to partially compensate, yet that may not be enough to prevent the probability of high effort from declining. Bénabou and Tirole (2003) note that if these considerations are not taken into account, the "outside observer might actually underestimate the power of these incentives [and] conclude that rewards are negative reinforcers."

Here, in contrast, the resolution to the puzzle is that the agent adjusts his effort at home when presented with $\omega^{\prime \prime}$ instead of $\omega^{\prime}$. The higher rewards at work leads the agent to work less hard at home. As labor income then plays a more significant role in the agent's overall well-being, weaker incentives are sufficient to induce him to work harder on the job. Interestingly, the optimal contract that implements a fixed $a_{1}$ depends on the principal's preferences over $a_{2}$.

Assume next that the principal does not care directly about $a_{2}$ and let $\underline{\omega}^{\prime}$ and $\underline{\omega}^{\prime \prime}$ denote the optimal (i.e. cheapest) way of inducing $a_{1}^{\prime}$ and $a_{1}^{\prime \prime}$, respectively, with $a_{1}^{\prime \prime}>a_{1}^{\prime}$. I will show that if marginal costs do not increase too quickly, then $M\left(a_{1}^{\prime \prime} \mid \underline{\omega}^{\prime \prime}\right)>M\left(a_{1}^{\prime} \mid \underline{\omega}^{\prime}\right)$ whereas $M^{\prime}\left(a_{1}^{\prime \prime} \mid \underline{\omega}^{\prime \prime}\right)<M^{\prime}\left(a_{1}^{\prime} \mid \underline{\omega}^{\prime}\right)$. While an outsider might explain the former by appealing to higher effort costs, the puzzle remains that the agent's utility appears to be less responsive to a marginal increase in effort under 
$\underline{\omega}^{\prime \prime}$. Again, the latter might be interpreted as indicating that explicit incentives are weaker when the agent works harder. As before, however, this ignores that the intensity with which the agent pursues private rewards has changed as well.

The most popular multi-tasking model is due to Holmström and Milgrom (1987, 1991). Owing to the specific functional forms that are imposed it is often referred to as the Linear-Exponential-Normal (LEN) model. For instance, contracts are restricted to be linear. Holmström and Milgrom (1991) examine certain settings in which the agent receives (deterministic) private rewards from pursuing tasks that do not yield contractible signals. While they discuss optimal contract design in these settings, they stop short of discussing intrinsic versus extrinsic motivation. In fact, it is arguably the case that the vast amount of structure that makes the LEN model so famously tractable inherently limits its suitability for studying some of the intricacies of private rewards. The slope of the linear contract is uniquely characterized by the incentive constraint that $a_{1}$ must be optimal for the agent. Given that this coefficient is typically interpreted as measuring the strength of incentives, the LEN model thus cannot deliver the more nuanced explanation of intrinsic motivation identified here. In fact, $a_{2}$ is uniquely determined by the $a_{1}$ value the principal implements. Finally, the LEN model is not rich enough to explain why the agent may earn economic rents.

Moreover, the LEN model overlooks some implications of common agency, as described in a companion paper, Kirkegaard (2015). Indeed, Kirkegaard (2014) documents that the LEN model's predictions are not robust even when there are no private rewards. Together, this trilogy of papers thus aims to contribute to an understanding of multi-tasking outside the confines of the LEN model. The two competing models of multi-tasking are briefly contrasted in Section 6 .

Section 2 provides an example. Sections 3 and 4 analyze the problem from the agent's and principal's perspective, respectively, culminating in a justification of the FOA. Technical extensions are discussed in Section 5. Section 6 focuses on intrinsic and extrinsic motivation in a particularly tractable version of the model. It is also established that the agent may earn economic rents. This section is largely self-contained. Section 7 compares and discusses the model's properties with those of the standard model. Section 8 concludes. 


\section{An example}

Actions and outcomes are continuous in the general model, but the following example assumes binary outcomes. The agent exerts effort $a_{1}$ on the job and $a_{2}$ on pursuing private rewards. The former produces a failure with probability $p_{1}\left(a_{1}\right)$ and a success with probability $1-p_{1}\left(a_{1}\right)$. The contract, $(\underline{w}, \bar{w})$, specifies pay in the two cases. Independently, $a_{2}$ yields a small private reward with probability $p_{2}\left(a_{2}\right)$ and a large reward with probability $1-p_{2}\left(a_{2}\right)$. The agent's utility is $m(w)$ if a small private reward is realized, where $m(w)$ is a negative, increasing, and concave function of labor income. For simplicity, the agent's utility is zero regardless of pay otherwise. Cost of effort is $c_{1} a_{1}+c_{2} a_{2}, c_{1}, c_{2}>0$, and expected utility is

$$
\left[m(\bar{w})-(m(\bar{w})-m(\underline{w})) p_{1}\left(a_{1}\right)\right] p_{2}\left(a_{2}\right)-c_{1} a_{1}-c_{2} a_{2} .
$$

Assume that $p_{1}\left(a_{1}\right)$ and $p_{2}\left(a_{2}\right)$ are strictly decreasing and strictly log-convex. Then, $p_{1}\left(a_{1}\right) p_{2}\left(a_{2}\right)$ is convex in $\left(a_{1}, a_{2}\right)$. Hence, the agent's problem is concave whenever $m(\bar{w}) \geq m(\underline{w})$. For any interior action that is to be induced, the agent's two first-order conditions determine $m(\underline{w})$ and $m(\bar{w})$ (assuming a solution exists),

$$
m(\underline{w})=\frac{c_{2}}{p_{2}^{\prime}\left(a_{2}\right)}+\frac{c_{1}\left(1-p_{1}\left(a_{1}\right)\right)}{p_{1}^{\prime}\left(a_{1}\right) p_{2}\left(a_{2}\right)}, m(\bar{w})=\frac{c_{2}}{p_{2}^{\prime}\left(a_{2}\right)}-\frac{c_{1} p_{1}\left(a_{1}\right)}{p_{1}^{\prime}\left(a_{1}\right) p_{2}\left(a_{2}\right)},
$$

with $m(\bar{w})>m(\underline{w})$, since $p_{1}^{\prime}\left(a_{1}\right)<0$. As long as the participation constraint is satisfied, a fixed $a_{1}$ can be implemented with a range of contracts that induce different $a_{2}$. As $p_{2}\left(a_{2}\right)$ is log-convex, $(i) m(\bar{w})$ and $m(\underline{w})$ are decreasing in $a_{2}$ whereas $(i i) m(\bar{w})-m(\underline{w})$ and $m(\bar{w}) / m(\underline{w})$ are increasing in $a_{2}$. Thus, there is an inverse relationship between $(i)$ wage levels and $(i i)$ "explicit incentives", holding $a_{1}$ fixed. Note that a principal who does not directly care about $a_{2}$ will induce the highest feasible $a_{2}$ value, forcing the participation constraint to bind. However, if he derives large disutility from $a_{2}$ it is better to induce a lower $a_{2}$ and concede rents to the agent. Section 6 establishes that in the former case $m(\bar{w})-(m(\bar{w})-m(\underline{w})) p_{1}\left(a_{1}\right)$ is increasing in $a_{1}$ but $-(m(\bar{w})-m(\underline{w})) p_{1}^{\prime}\left(a_{1}\right)$ is decreasing in $a_{1}$. Hence, an outsider who thinks $a_{2}$ is exogenous would conclude that the agent's utility from wages are higher, but explicit incentives weaker, the harder he is induced to work. 


\section{The agent's problem}

Before describing the model I briefly preview some of the key steps to justifying the FOA with private rewards. Kirkegaard's (2014) multi-task justifications of the FOA is a good starting point. In particular, one of his justifications apply to environments in which the optimal contract turns out to be monotonic and such that the outcomes of the two tasks are substitutes from the agent's point of view. That is, a marginal improvement in the performance of task 2 is worth less to the agent if he performed extremely well on task 1. However, in Kirkegaard (2014) the principal rewards both tasks. On the other hand, in the present setting it is quite natural to assume that labor income and private rewards are substitutes. That is, private rewards yields substitutability essentially for free. However, it turns out to be substantially harder to establish monotonicity.

To establish monotonicity, a main challenge is to sign the multipliers of the incentive compatibility constraints. This is accomplished by extending a classic argument by Rogerson (1985), involving a doubly-relaxed maximization problem. In essence, Rogerson (1985) shows that it is sufficient to prevent the agent from working less hard than intended. In the present setting, however, there are two tasks. As is perhaps intuitive, it turns out to be sufficient to simultaneously prevent the agent from shirking on job and working too hard on the private task.

Thus, the following assumptions on the primitives (technology and preferences) are used to either establish monotonicity and substitutability or to prove that the FOA is valid whenever the candidate contract takes such a form.

I consider a relatively simple model of a principal-agent relationship with endogenous private rewards. The agent performs two "tasks", $a_{1}$ and $a_{2}$, each of which belong to a compact interval, $a_{i} \in\left[\underline{a}_{i}, \bar{a}_{i}\right], i=1,2$. The first task captures the agent's effort on the job, as a result of which a contractible signal, $x_{1}$, is produced. The signal's marginal distribution is $G^{1}\left(x_{1} \mid a_{1}\right)$. The second "task" reflects the agent's pursuit of a private reward. The agent receives a (possibly non-monetary) reward, $x_{2}$, which is determined by the marginal distribution function $G^{2}\left(x_{2} \mid a_{2}\right)$. Here, $a_{2}$ could measure life-style choices and $x_{2}$ the health outcome. Assume $x_{i}$ belongs to a compact interval, $\left[\underline{x}_{i}, \bar{x}_{i}\right]$, which is independent of $a_{i}$. Assume $G^{1}$ and $G^{2}$ are continuously differentiable in both variables 
to the requisite degree. Let $g^{1}\left(x_{1} \mid a_{1}\right)$ and $g^{2}\left(x_{2} \mid a_{2}\right)$ denote the respective densities. Assume $g^{i}\left(x_{i} \mid a_{i}\right)>0$ for all $x_{i} \in\left[\underline{x}_{i}, \bar{x}_{i}\right]$ and all $a_{i} \in\left[\underline{a}_{i}, \bar{a}_{i}\right]{ }^{6}$. Note that each marginal distribution depends only on one task. ${ }^{7}$ This property is further strengthened by assuming that $x_{1}$ and $x_{2}$ are independent.

Assumption A1 (Independence): Outcomes are independent, i.e. given $a_{1}$ and $a_{2}$, the joint distribution is given by

$$
F\left(x_{1}, x_{2} \mid a_{1}, a_{2}\right)=G^{1}\left(x_{1} \mid a_{1}\right) G^{2}\left(x_{2} \mid a_{2}\right)
$$

More structure is required on the components of the joint distribution function. Thus, define $l^{i}\left(x_{i} \mid a_{i}\right)=\ln g^{i}\left(x_{i} \mid a_{i}\right)$ and let $l_{a_{i}}^{i}\left(x_{i} \mid a_{i}\right)$ denote the likelihoodratio, i.e. the derivative of $l^{i}\left(x_{i} \mid a_{i}\right)$ with respect to $a_{i}, i=1,2$.

Assumption A2 (MLRP): The marginal distributions have the monotone likelihood ratio property, i.e. for all $a_{i} \in\left[\underline{a}_{i}, \bar{a}_{i}\right]$ it holds that

$$
\frac{\partial}{\partial x_{i}}\left(l_{a_{i}}^{i}\left(x_{i} \mid a_{i}\right)\right)=\frac{\partial^{2} \ln g^{i}\left(x_{i} \mid a_{i}\right)}{\partial a_{i} \partial x_{i}} \geq 0 \text { for all } x_{i} \in\left[\underline{x}_{i}, \bar{x}_{i}\right]
$$

with strict inequality on a subset of strictly positive measure, $i=1,2$.

Assumption A2 implies that $G_{a_{i}}^{i}\left(x_{i} \mid a_{i}\right)<0$ for all $x_{i} \in\left(\underline{x}_{i}, \bar{x}_{i}\right){ }^{8}$ The interpretation is that when the agent works harder, bad outcomes are less likely. In particular, if $a_{i}^{\prime}>a_{i}^{\prime \prime}$ then $G^{i}\left(x_{i} \mid a_{i}^{\prime}\right)$ first order stochastically dominates $G^{i}\left(x_{i} \mid a_{i}^{\prime}\right){ }^{9}$

It is assumed that $x_{1}$ and $x_{2}$ are realized at the same time. In an important paper, Rogerson (1985) justifies the FOA in a one-signal, one-task model. He

\footnotetext{
${ }^{6}$ Throughout, all exogenous functions are assumed continously differentiable to the requisite
} degree. For brevity, statements to that effect are omitted from the numbered assumptions.

${ }^{7}$ This is somewhat less restrictive than it appears at first glance. For instance, assume $G_{i}$ is a one-parameter distribution, and that $a_{1}$ and $a_{2}$ both influence the parameter. That is, $G_{i}$ can be written $G_{i}\left(x_{i} \mid t_{i}\left(a_{1}, a_{2}\right)\right)$. In this case, the problem can simply be reformulated to make $t_{1}$ and $t_{2}$ the two choice variables. However, the possibility that $a_{1}$ and $a_{2}$ influence different parameters of one or both of the marginal distributions is ruled out.

${ }^{8}$ To see this, recall first that the expected value of $l_{a_{i}}^{i}\left(x_{i} \mid a_{i}\right)$ is zero. Assumption A2 therefore implies that $l_{a_{i}}^{i}\left(\underline{x}_{i} \mid a_{i}\right)<0<l_{a_{i}}^{i}\left(\bar{x}_{i} \mid a_{i}\right)$. Since $G_{a_{i}}^{i}\left(\underline{x}_{i} \mid a_{i}\right)=G_{a_{i}}^{i}\left(\bar{x}_{i} \mid a_{i}\right)=0$, it follows that $G_{a_{i}}^{i}\left(x_{i} \mid a_{i}\right)=\int_{\underline{x}_{i}}^{x_{i}} l_{a_{i}}^{i}\left(z_{i} \mid a_{i}\right) g^{i}\left(x_{i} \mid a_{i}\right)<0$ for all $x_{i} \in\left(\underline{x}_{i}, \bar{x}_{i}\right)$.

${ }^{9}$ The model would reduce to the standard single-task, one-signal model if $G^{2}\left(x_{2} \mid a_{2}\right)$ was degenerate and independent of $a_{2}$. 
assumes the distribution function satisfies MLRP and that it is convex in the (one-dimensional) action. Rogerson (1985) refers to the latter as the convexity of distribution function condition (CDFC). Kirkegaard (2014) extends the justification of the FOA to allow multiple tasks and signals. He shows that a natural extension of the CDFC is to assume that the distribution function is convex in the (now many-dimensional) action. The same assumption is imposed here.

Assumption A3 (LOCC): $F\left(x_{1}, x_{2} \mid a_{1}, a_{2}\right)$ satisfies the lower orthant convexity condition; $F\left(x_{1}, x_{2} \mid a_{1}, a_{2}\right)$ is weakly convex in $\left(a_{1}, a_{2}\right)$ for all $\left(x_{1}, x_{2}\right)$ and $\left(a_{1}, a_{2}\right)$.

Assumption A3 necessitates that $G^{i}$ is convex in $a_{i}, i=1,2$. In fact, it implies that $G_{a_{i} a_{i}}\left(x_{i} \mid a_{i}\right)>0$ for all $x_{i} \in\left(\underline{x}_{i}, \bar{x}_{i}\right){ }^{10}$ A sufficient condition for LOCC is that $G^{1}$ and $G^{2}$ are both log-convex. Kirkegaard (2014) lists several examples. See also Ábrahám et al (2011), discussed in more detail at the end of this section. Alternatively, fix some $G^{1}$ that is strictly convex in $a_{1}$, but not necessarily logconvex. Then, there is always some "sufficiently convex" $G^{2}$ function that ensures that Assumption A3 is satisfied. For example, a non-negative function $h(z)$ is said to be $\rho$-convex if $h(z)^{\rho} / \rho$ is convex, or $h^{\prime \prime}(z) h(z) / h^{\prime}(z)^{2} \geq 1-\rho$ for all $z$. Thus, a $\rho$-convex function is log-convex if and only if $\rho \leq 0$ (and convex if and only if $\rho \leq 1)$. It is easy to see that if $G^{2}\left(x_{2} \mid a_{2}\right)$ satisfies Assumption A2 and is $\rho$-convex in $a_{2}$ (for all $x_{2}$ ) for some small enough $\rho$ (i.e. $\rho$ is negative, but numerically large), then Assumption A3 is satisfied. ${ }^{11}$ To reiterate, as long as $G^{1}$ satisfies a strict version of CDFC there are $G^{2}$ functions that will permit the FOA to be justified even when allowing for private rewards.

Assumptions A1-A3 describes the "technology". The next set of assumptions describes the agent's preferences. Given action $\left(a_{1}, a_{2}\right)$, wage $w$, and private reward $x_{2}$, the agent's utility is assumed to take the form

$$
v\left(w, x_{2}\right)-c\left(a_{1}, a_{2}\right),
$$

\footnotetext{
${ }^{10}$ LOCC necessitates that $G_{a_{i} a_{i}}^{i} \geq 0$ and $G^{1} G^{2} G_{a_{1} a_{1}}^{1} G_{a_{2} a_{2}}^{2}-\left(G_{a_{1}}^{1} G_{a_{2}}^{2}\right)^{2} \geq 0$. At any interior $\left(x_{1}, x_{2}\right)$, the last term is strictly positive, by A2. Thus, $G_{a_{1} a_{1}}^{1}>0$ and $G_{a_{2} a_{2}}^{2}>0$ are necessary.

${ }^{11}$ The inequality in the previous footnote can be written $G^{1} G_{a_{1} a_{1}}^{1}\left(G^{2} G_{a_{2} a_{2}}^{2} /\left(G_{a_{2}}^{2}\right)^{2}\right)$ $\left(G_{a_{1}}^{1}\right)^{2} \geq 0$, for interior $\left(x_{1}, x_{2}\right)$. By $\rho$-convexity, the left hand side is greater than $G^{1} G_{a_{1} a_{1}}^{1}(1-\rho)-\left(G_{a_{1}}^{1}\right)^{2} \geq 0$. Hence, the inequality is satisfied if $\rho$ is small enough.
} 
where $v$ is a benefit function and $c$ a cost function. Both functions are assumed to be continuously differentiable in both their arguments to the requisite degree. The function $v\left(w, x_{2}\right)$ is strictly increasing and strictly concave in both arguments, $v_{i}>0>v_{i i}, i=1,2$, where subscripts denote derivatives. The cost function is likewise assumed to be strictly increasing. It is also assumed to be convex in $\left(a_{1}, a_{2}\right)$. While Assumption A1 imply that there is no stochastic interaction between $a_{1}$ and $a_{2}$, the cost function allows interaction between tasks.

Note that if the private reward, $x_{2}$, is income, then $v\left(w, x_{2}\right)$ could be written $v\left(w+x_{2}\right)$, in which case it is automatic that $v_{12}<0$. That is, employment income and outside income are substitutes. Indeed, even when $x_{2}$ is not income it is natural to assume that $w$ and $x_{2}$ are strict substitutes. Thus, it will be assumed that $v_{12}<0$; the higher $x_{2}$ is, the lower is the marginal utility of additional employment income. I will also assume that $a_{1}$ and $a_{2}$ are weak substitutes in the cost function, or $c_{12} \geq 0$. That is, the marginal cost of increasing $a_{1}$ is higher the higher $a_{2}$ is. Assumption A4 summarizes these assumptions.

Assumption A4 (Substitutes): The agent's Bernoulli utility is $v\left(w, x_{2}\right)$ $c\left(a_{1}, a_{2}\right) ; v\left(w, x_{2}\right)$ is strictly increasing and strictly concave in both $w$ and $x_{2}$, while $c\left(a_{1}, a_{2}\right)$ is strictly increasing and weakly convex in $\left(a_{1}, a_{2}\right)$. The rewards $w$ and $x_{2}$ are strict substitutes; $v_{12}\left(w, x_{2}\right)<0$. The tasks are weak substitutes; $c_{12}\left(a_{1}, a_{2}\right) \geq 0$.

The principal specifies a contract of the form $w\left(x_{1}\right)$. That is, the contract details the wage to the agent if the verifiable signal is $x_{1} \cdot{ }^{12}$ Upon taking action $\left(a_{1}, a_{2}\right)$, the agent's expected payoff is then

$$
E U\left(a_{1}, a_{2}\right)=\iint v\left(w\left(x_{1}\right), x_{2}\right) g^{1}\left(x_{1} \mid a_{1}\right) g^{2}\left(x_{2} \mid a_{2}\right) d x_{1} d x_{2}-c\left(a_{1}, a_{2}\right)
$$

For notational simplicity, $E U\left(a_{1}, a_{2}\right)$ suppresses the dependency on the contract. Imagine the principal's intention is to induce the agent to take action $\left(a_{1}^{\prime}, a_{2}^{\prime}\right)$. For the agent to comply, $E U\left(a_{1}, a_{2}\right)$ must be maximized at $\left(a_{1}^{\prime}, a_{2}^{\prime}\right)$. Assuming

\footnotetext{
${ }^{12}$ This ignores the possibility that the principal may ask the agent to report $x_{2}$ and then make the wage dependent upon both $x_{1}$ and the report. It can easily be verified that the principal can never gain from such a scheme in the special version of the model in Section 6 .
} 
the action is interior, this at the very least necessitates that expected utility is at a stationary point at $\left(a_{1}^{\prime}, a_{2}^{\prime}\right)$, or $E U_{1}\left(a_{1}^{\prime}, a_{2}^{\prime}\right)=E U_{2}\left(a_{1}^{\prime}, a_{2}^{\prime}\right)=0$. The FOA relies on the latter conditions being not only necessary but also sufficient for utility maximization. To this end, the typical approach is to establish that $E U\left(a_{1}, a_{2}\right)$ is concave, although Kirkegaard (2014) uses a somewhat different method.

If $w\left(x_{1}\right)$ is non-decreasing in $x_{1}$, then, given Assumption $\mathrm{A} 4, v\left(w\left(x_{1}\right), x_{2}\right)$ is increasing in $x_{1}$ and $x_{2}$, and submodular in the two. Now, Kirkegaard (2014) proves that if the agent faces such a reward function, then the FOA is valid if LOCC (Assumption A3) is satisfied as well. ${ }^{13}$ In fact, $E U\left(a_{1}, a_{2}\right)$ is concave in $\left(a_{1}, a_{2}\right)$. To see this, note that after integration by parts with respect to $x_{2}$,

$E U\left(a_{1}, a_{2}\right)=\int\left(v\left(w\left(x_{1}\right), \bar{x}_{2}\right)-\int v_{2}\left(w\left(x_{1}\right), x_{2}\right) G^{2}\left(x_{2} \mid a_{2}\right) d x_{2}\right) g^{1}\left(x_{1} \mid a_{1}\right) d x_{1}-c\left(a_{1}, a_{2}\right)$

Assuming for simplicity that $w\left(x_{1}\right)$ is differentiable (it will later be established that the optimal contract is indeed differentiable), another round of integrating by parts, this time with respect to $x_{1}$, yields

$$
\begin{aligned}
E U\left(a_{1}, a_{2}\right)= & v\left(w\left(\bar{x}_{1}\right), \bar{x}_{2}\right)-\int v_{1}\left(w\left(x_{1}\right), \bar{x}_{2}\right) w^{\prime}\left(x_{1}\right) G^{1}\left(x_{1} \mid a_{1}\right) d x_{1} \\
& +\iint v_{12}\left(w\left(x_{1}\right), x_{2}\right) w^{\prime}\left(x_{1}\right) G^{1}\left(x_{1} \mid a_{1}\right) G^{2}\left(x_{2} \mid a_{2}\right) d x_{1} d x_{2} \\
& -\int v_{2}\left(w\left(\bar{x}_{1}\right), x_{2}\right) G^{2}\left(x_{2} \mid a_{2}\right) d x_{2}-c\left(a_{1}, a_{2}\right)
\end{aligned}
$$

Recall that $v_{1}, v_{2}>0 \geq v_{12}$ while $G^{1}\left(x_{1} \mid a_{1}\right), G^{2}\left(x_{2} \mid a_{2}\right), G^{1}\left(x_{1} \mid a_{1}\right) G^{2}\left(y_{2} \mid a_{2}\right)$, and $c\left(a_{1}, a_{2}\right)$ are all convex in $\left(a_{1}, a_{2}\right)$. Thus, as long as $w^{\prime}\left(x_{1}\right) \geq 0, E U\left(a_{1}, a_{2}\right)$ is the sum of concave functions. The first Lemma records this fact.

Lemma 1 Assume $w^{\prime}\left(x_{1}\right) \geq 0$ for all $x_{1} \in\left[\underline{x}_{1}, \bar{x}_{1}\right]$ and that Assumptions A1-A4 hold. Then, the agent's expected utility, EU $\left(a_{1}, a_{2}\right)$, is concave in $\left(a_{1}, a_{2}\right)$.

\footnotetext{
${ }^{13}$ Kirkegaard (2014) uses insights from choice under uncertainty to explain the intuition underlying this result. Jewitt (1988) and Conlon (2009) present two results with a similar flavour in a model with two signals but a single task. Kirkegaard's (2014) characterization is more general, as it extends to more signals and more tasks. For instance, he identifies the appropriate generalization of submodularity when there are more than two signals.
} 
Unfortunately, it is far from trivial to establish that $w\left(x_{1}\right)$ is non-decreasing. Thus, the following analysis is primarily devoted to that particular problem.

First, however, note that Lemma 1 implies that $E U\left(a_{1}, a_{2}\right)$ must be concave in $a_{1}$ and concave in $a_{2}$, whenever $w^{\prime}\left(x_{1}\right) \geq 0$. In fact, (3) implies that $E U_{22}\left(a_{1}, a_{2}\right)<0$ as $G_{a_{2} a_{2}}^{2}>0$. The expression in (4) also makes it clear that the last two parts of Assumption A4 pull in the same direction. In particular, the two tasks are strict substitutes in the agent's expected utility, or $E U_{12}\left(a_{1}, a_{2}\right) \leq 0$, whenever $w^{\prime}\left(x_{1}\right) \geq 0$. Indeed, if $w^{\prime}\left(x_{1}\right)>0$ on a subset of positive measure, then $v_{12}<0$ and $c_{12} \geq 0$ imply that $E U_{12}\left(a_{1}, a_{2}\right)<0$.

To proceed, it is necessary to impose more specific assumptions on the agent's risk preferences over labor income, $w$. Thus, it will be assumed that the absolute risk aversion with respect to $w$ is decreasing in $x_{2}$. In other words, the agent is less sensitive to risk in labor income the higher the private reward is.

Assumption A5 (Decreasing absolute risk aversion): The agent's absolute risk aversion over labor income is decreasing in $x_{2}$. That is, $v_{1}\left(w, x_{2}\right)$ is log-supermodular in $\left(w, x_{2}\right)$, or

$$
\frac{\partial^{2} \ln v_{1}\left(w, x_{2}\right)}{\partial w \partial x_{2}} \geq 0 \text { for all } w \text { and all } x_{2} \in\left[\underline{x}_{2}, \bar{x}_{2}\right] .
$$

Of course, (5) is equivalent to

$$
\frac{\partial}{\partial x_{2}}\left(\frac{-v_{11}\left(w, x_{2}\right)}{v_{1}\left(w, x_{2}\right)}\right) \leq 0 .
$$

For future reference, note that Assumption A2 (MLRP) is equivalent to the requirement that $g^{i}\left(x_{i} \mid a_{i}\right)$ is log-supermodular in $\left(x_{i}, a_{i}\right), i=1,2$. Next, let

$$
V\left(w, a_{2}\right)=\int v\left(w, x_{2}\right) g^{2}\left(x_{2} \mid a_{2}\right) d x_{2},
$$

such that the agent's expected utility can be written as

$$
E U\left(a_{1}, a_{2}\right)=\int V\left(w\left(x_{1}\right), a_{2}\right) g^{1}\left(x_{1} \mid a_{1}\right) d x_{1}-c\left(a_{1}, a_{2}\right) .
$$


Given (6), note that

$$
\begin{aligned}
V_{1}\left(w, a_{2}\right) & =\int v_{1}\left(w, x_{2}\right) g^{2}\left(x_{2} \mid a_{2}\right) d x_{2}>0, \text { and } \\
V_{12}\left(w, a_{2}\right) & =\int v_{1}\left(w, x_{2}\right) g_{a_{2}}^{2}\left(x_{2} \mid a_{2}\right) d x_{2}<0 .
\end{aligned}
$$

Here, $V_{1}\left(w, a_{2}\right)$ describes the expected marginal utility of additional labor income given the agent's effort on the private task is $a_{2}$. Of course, $V_{12}\left(w, a_{2}\right)$ captures how this expectation changes with $a_{2}$. Assumptions A2 and A4 together implies that $V_{12}\left(w, a_{2}\right)<0$. Evidently, $V_{1}\left(w, a_{2}\right)$ is strictly decreasing in $w$, or $V_{11}\left(w, a_{2}\right)<0$. Moreover, the term under the integration sign in $V_{1}\left(w, a_{2}\right)$ is, by Assumptions $\mathrm{A} 2$ and $\mathrm{A} 5$, log-supermodular in $\left(w, x_{2}, a_{2}\right)$. As described by e.g. Athey (2002), log-supermodularity is preserved under integration. Thus, $V_{1}\left(w, a_{2}\right)$ is log-supermodular in $\left(w, a_{2}\right)$. That is, the agent's decreasing absolute risk aversion aggregates, or carries over to the expected utility in (6). Hence,

$$
\frac{\partial}{\partial a_{2}}\left(\frac{-V_{11}\left(w, a_{2}\right)}{V_{1}\left(w, a_{2}\right)}\right) \leq 0
$$

such that the agent is less sensitive to risk in labor income the better the distribution of private rewards is. Equivalently,

$$
\frac{\partial}{\partial w}\left(\frac{-V_{12}\left(w, a_{2}\right)}{V_{1}\left(w, a_{2}\right)}\right) \leq 0
$$

The latter property is especially important. Technically, the assumption that $g^{2}\left(x_{2} \mid a_{2}\right)$ is $\log$-supermodular can be relaxed (although $G_{a_{2}}^{2} \leq 0$ is still required) if the assumption that (5) holds is replaced by the assumption that (7) holds. For instance, if $x_{2}$ is income and $v\left(w, x_{2}\right)=-e^{-r\left(w+x_{2}\right)}, r>0$, then the agent exhibits constant absolute risk aversion in total income (and its components). In this case, (7) is trivially satisfied for any $g^{2}\left(x_{2} \mid a_{2}\right)$.

At this point, it is instructive to compare the present set-up with the literature on hidden savings. Ábrahám et al (2011) consider a situation where the agent works for the principal while simultaneously privately investing in a risk-free asset. Performance on the job, $x_{1}$, is thus the only source of uncertainty. Ábrahám et al (2011) justify the FOA by assuming that the distribution of $x_{1}$ is log-convex 
in effort and that the agent has decreasing absolute risk aversion. These two assumptions can be thought of as special cases of Assumptions A3 and A5 in the current paper. Here, rewards are both uncertain and possibly non-monetary.

To complete the description of the agent's problem, assume that the only constraint other than incentive compatibility is a participation constraint. That is, the agent must earn expected utility of at least $\bar{u}$ to sign the contract.

\section{Contracts with private rewards}

The previous section describes the problem from the agent's point of view. Consider now the principal's problem. First, assume that the principal is risk neutral. Let $B\left(a_{1}, a_{2}\right)$ denote the principal's direct benefit of the agent's action and assume that it is continuously differentiable. For instance, $B\left(a_{1}, a_{2}\right)$ could be the expected value of $x_{1}$, given $a_{1}$. As explained below, for technical reasons I assume that $B_{2}\left(a_{1}, a_{2}\right) \leq 0$, such that the principal prefers $a_{2}$ to be as small as possible. This assumption is of course satisfied if $B$ is independent of $a_{2}$. In the more specialized model in Section 6 , it is possible to allow $B_{2}\left(a_{1}, a_{2}\right)>0$, however. Finally, let $E\left[w \mid a_{1}, a_{2}\right]$ denote the expected wage costs if the agent is induced to take action $\left(a_{1}, a_{2}\right)$.

Assumption A6 (The principal's preferences): The principal is risk neutral, with expected utility $B\left(a_{1}, a_{2}\right)-E\left[w \mid a_{1}, a_{2}\right]$, where $B_{2}\left(a_{1}, a_{2}\right) \leq 0$ for all $\left(a_{1}, a_{2}\right)$.

It is natural to assume that $B\left(a_{1}, a_{2}\right)$ is increasing in $a_{1}$. Indeed, Proposition 2 in Section 7.2 will establish that if it is optimal to implement an interior action, then $B_{1}>0$ at that point. However, $B$ need not be globally increasing in $a_{1}$.

The principal's problem is to maximize $B\left(a_{1}, a_{2}\right)$ less wage costs, subject to individual rationality and incentive compatibility, or

$$
\begin{gathered}
\max _{a_{1}, a_{2}, w} B\left(a_{1}, a_{2}\right)-\int w\left(x_{1}\right) g_{1}\left(x_{1} \mid a_{1}\right) d x_{1} \\
\text { st. } \quad E U\left(a_{1}, a_{2}\right) \geq \bar{u} \\
\left(a_{1}, a_{2}\right) \in \arg \max _{\left(a_{1}^{\prime}, a_{2}^{\prime}\right) \in\left[\underline{a}_{1}, \bar{a}_{1}\right] \times\left[\underline{a}_{2}, \bar{a}_{2}\right]} E U\left(a_{1}^{\prime}, a_{2}^{\prime}\right)
\end{gathered}
$$


Any action (if one exists) that solves the problem is henceforth referred to as a second-best action.

It is important to realize that the contract indirectly determines not only how hard the agent works for the principal, but also how hard he works on the private task. From the agent's point of view, the function $v\left(w\left(x_{1}\right), x_{2}\right)$ is crucial to the decision of how much effort to devote to each task. It is immaterial that the reward $x_{2}$ happens to be not paid by the principal.

Assume the principal wishes to induce an interior action. Then, as mentioned previously, it is necessary that $E U$ achieves a stationary point at the targeted action, or $E U_{1}\left(a_{1}, a_{2}\right)=0=E U_{2}\left(a_{1}, a_{2}\right)$. These constraints are referred to as the "local" incentive compatibility constraints. As in the existing FOA literature, the main objective of this part of the paper is to establish conditions under which the local constraints are in fact sufficient for "global" incentive compatibility.

Consider the following relaxed problem, so named because the incentive compatibility constraint in the original problem has been relaxed,

$$
\begin{aligned}
\max _{a_{1}, a_{2}, w} B\left(a_{1}, a_{2}\right)-\int w\left(x_{1}\right) g_{1}\left(x_{1} \mid a_{1}\right) d x_{1} \\
\text { st. } \quad E U\left(a_{1}, a_{2}\right) \geq \bar{u} \\
E U_{1}\left(a_{1}, a_{2}\right)=0 \\
E U_{2}\left(a_{1}, a_{2}\right)=0
\end{aligned}
$$

The FOA is said to be valid if the solution to the relaxed problem also solves the unrelaxed (original) problem.

Let $\lambda \geq 0$ denote the multiplier to the participation constraint, and $\mu_{1}$ and $\mu_{2}$ denote the multipliers to the two local incentive compatibility constraints in the relaxed problem. Assuming interior wages, the optimal wage if $x_{1}$ is observed is implicitly characterized by the necessary first order condition,

$$
V_{1}\left(w, a_{2}\right)\left[\lambda+\mu_{1} l_{a_{1}}^{1}\left(x_{1} \mid a_{1}\right)\right]+\mu_{2} V_{12}\left(w, a_{2}\right)=1
$$

or

$$
\lambda+\mu_{1} l_{a_{1}}^{1}\left(x_{1} \mid a_{1}\right)=\frac{1}{V_{1}\left(w, a_{2}\right)}-\mu_{2} \frac{V_{12}\left(w, a_{2}\right)}{V_{1}\left(w, a_{2}\right)} .
$$


Qualitatively, the solution almost certainly depends on the sign of the two multipliers $\mu_{1}$ and $\mu_{2}$. Indeed, it is not even clear that there is a unique solution. However, later arguments will establish that it is sufficient to focus on multipliers for which $\mu_{1} \geq 0 \geq \mu_{2}$. Then, the contract $w\left(x_{1}\right)$ is well-behaved.

Lemma 2 Given Assumptions A1-A6 and interior wages, $w\left(x_{1}\right)$ as defined in (9) is unique whenever $\mu_{1} \geq 0 \geq \mu_{2}$. Moreover, the solution is differentiable, with $w^{\prime}\left(x_{1}\right) \geq 0$ for all $x_{1} \in\left[\underline{x}_{1}, \bar{x}_{1}\right]$. If $\mu_{1}>0 \geq \mu_{2}$, then $w^{\prime}\left(x_{1}\right)>0$ for a subset of $\left[\underline{x}_{1}, \bar{x}_{1}\right]$ of strictly positive measure.

Proof. Given $\mu_{2} \leq 0, V_{11}<0$ and (7) imply that the right hand side of (9) is strictly increasing in $w$. Thus, for each $x_{1}$ there is at most one solution to (9), $w\left(x_{1}\right)$. Differentiability then follows automatically from the differentiability of all the components in (9). Since $\mu_{1} \geq 0$, Assumption A2 (MLRP) implies that the left hand side is non-decreasing in $x_{1}$. Hence, $w\left(x_{1}\right)$ is non-decreasing in $x_{1}$. The last part likewise follows from Assumption A2.

The next step utilizes Rogerson's (1985) idea of considering a doubly-relaxed problem. In Rogerson's one-task model, the relaxed incentive compatibility constraint, $E U_{1}=0$, is replaced with the even weaker constraint that $E U_{1} \geq 0$. In the current multi-task model, the appropriate doubly-relaxed problem assumes $E U_{1} \geq 0$ and $E U_{2} \leq 0$, respectively. The set of feasible contracts (i.e. the constraint set) is obviously larger in the doubly-relaxed problem than in the relaxed problem. For interior actions, any contract that is incentive compatible (i.e. feasible in the unrelaxed problem) is also feasible in both the relaxed and doubly-relaxed problems. However, this does not hold for all incentive compatible contracts that induce boundary actions. For this reason, extra care must be taken in dealing with corner solutions.

Conveniently, $\mu_{1} \geq 0 \geq \mu_{2}$ must hold in the doubly-relaxed problem. As in Rogerson, assume there is a solution to the doubly-relaxed problem. In particular, this requires the constraint set to be non-empty. Likewise, for simplicity, it will be assumed that wages are interior. Rogerson impose assumptions directly on the utility functions (see his assumption A3-A4 and A6-A7) to achieve this.

Assumption A7 (The doubly-relaxed problem): A solution to the doublyrelaxed problem exists. Any solution involves only wages in the interior of the 
domain of $v\left(w, x_{2}\right)$.

Any solution to the doubly-relaxed problem must take the form in (9). By Lemma 2, any solution thus features non-decreasing wages. By Lemma 1, the agent's problem is concave. The contract is then incentive compatible if $E U_{1}=$ $E U_{2}=0$ at the intended action. Of course, the latter holds if $\mu_{1}>0>\mu_{2}$. Let $\left(a_{1}^{*}, a_{2}^{*}\right)$ denote an action that forms part of a solution to the double-relaxed problem.

Lemma 3 Given Assumptions A1-A7, any solution to the doubly-relaxed problem is incentive compatible and thus feasible in the unrelaxed problem. Moreover, if $a_{2}^{*}>\underline{a}_{2}$ then it is also feasible in the relaxed problem, which it also solves.

Proof. Wages are constant if $\mu_{1}=0$. Then, $E U_{1}<0$, which violates the doublyrelaxed constraints. Hence, $\mu_{1}>0$ and so $E U_{1}\left(a_{1}^{*}, a_{2}^{*}\right)=0$. Now, if $a_{2}^{*}$ is interior it must satisfy the first-order condition that

$$
\left[B_{2}\left(a_{1}, a_{2}\right)+\lambda E U_{2}\left(a_{1}^{*}, a_{2}^{*}\right)+\mu_{1} E U_{12}\left(a_{1}^{*}, a_{2}^{*}\right)\right]+\mu_{2} E U_{22}\left(a_{1}^{*}, a_{2}^{*}\right)=0 .
$$

By Assumption A6, $B_{2}\left(a_{1}, a_{2}\right) \leq 0$. Following the argument described after Lemma 1, it holds that $E U_{12}\left(a_{1}^{*}, a_{2}^{*}\right)<0$ given the properties of $w\left(x_{1}\right)$ described in Lemma 2 when $\mu_{1}>0 \geq \mu_{2}{ }^{14}$ Since $\lambda E U_{2}\left(a_{1}^{*}, a_{2}^{*}\right) \leq 0$, the term in the bracket in (10) is thus strictly negative. As $E U_{22}\left(a_{1}^{*}, a_{2}^{*}\right)<0$, it is therefore necessary that $\mu_{2}<0$. Hence, $E U_{2}\left(a_{1}^{*}, a_{2}^{*}\right)=0$. A similar argument applies if $a_{2}^{*}=\bar{a}_{2}$. Thus, both incentive constraints are binding and, by concavity, the agent's utility is maximized at $\left(a_{1}^{*}, a_{2}^{*}\right)$. That is, the contract is incentive compatible. If $a_{2}^{*}=\underline{a}_{2}$, it cannot be ruled out that $E U_{2}\left(a_{1}^{*}, a_{2}^{*}\right)<0$. Nevertheless, by concavity, such a solution on the boundary is still incentive compatible. This completes the proof of the first part of the Lemma. Finally, note that when $a_{2}^{*}>\underline{a}_{2}$, the solution is feasible in the relaxed problem. Since the constraint set is smaller in the relaxed problem, the last part of the Lemma follows.

\footnotetext{
${ }^{14}$ Note that Assumption A4 rules out that $v_{12}=c_{12}=0$. However, this case seems relatively uninteresting, as it would imply that there is a fixed $a_{2}$ which is optimal for the agent regardless of the contract. In particular, the last term in (8) would disappear. It is then easy to show that the FOA is valid if $G^{1}\left(x_{1} \mid a_{1}\right)$ satisfies CDFC.
} 
Lemma 3 is key to establishing the validity of the FOA. As explained after Lemma 2, the possibility of a corner solution gives rise to some complications. Thus, following the literature, assume the second-best action is interior. Then, the solution to the unrelaxed problem must satisfy $E U_{1}=E U_{2}=0$, which implies that it is feasible in the doubly-relaxed problem. By Lemma 3, however, the solution to the doubly-relaxed problem is in turn feasible in the unrelaxed problem. Hence, the solutions to the unrelaxed and doubly-relaxed problems coincide. Finally, Lemma 3 implies that as the solution to the doubly-relaxed problem involves an interior action, the relaxed problem identifies the exact same solution.

Theorem 1 Assume any second-best action $\left(a_{1}, a_{2}\right)$ is interior. Then, given Assumptions A1-A\%, the FOA is valid.

It is perhaps natural to question the assumption that it is optimal to induce the agent to work on the private task ( $a_{2}$ is interior). However, note that

$$
E U_{2}\left(a_{1}, \underline{a}_{2}\right)=\int\left(\int v\left(w\left(x_{1}\right), x_{2}\right) g_{a_{2}}^{2}\left(x_{2} \mid \underline{a}_{2}\right) d x_{2}\right) g^{1}\left(x_{1} \mid a_{1}\right) d x_{1}-c_{2}\left(a_{1}, \underline{a}_{2}\right) .
$$

The inner integral is strictly positive regardless of the contract. Thus, if $c_{2}\left(a_{1}, \underline{a}_{2}\right)=$ 0 , then $E U_{2}\left(a_{1}, \underline{a}_{2}\right)>0$ for any contract. In this case, it is impossible to persuade the agent to not pursue private rewards. Moreover, it is established in Section 6 that an interior $a_{2}$ is optimal in the multiplicative model.

\section{Extension: Relaxing LOCC}

The stringency of Rogerson's CDFC is the main source of criticism of the FOA in the standard model. As mentioned earlier, Assumption A3 (LOCC) generalizes CDFC to allow multi-tasking. In this section, I consider two possible ways of relaxing Assumption A3. The first requires mild assumptions on the marginal utility of labor income. The second extension requires more specific structure on the functional form of $v\left(w, x_{2}\right)$. 


\subsection{Supermodular marginal utility}

Jewitt (1988) was first to relax the CDFC. In the one-signal, one-task case, he replaces the CDFC assumption with the assumption that

$$
\int_{\underline{x}_{1}}^{x_{1}} G^{1}\left(y_{1} \mid a_{1}\right) d y_{1}
$$

is convex in $a_{1}$ for all $x_{1}$. Jewitt (1988) shows that this condition is sufficient to justify the FOA provided that the agent's utility is increasing and concave in $x_{1}$. Concavity must thus be established. In Jewitt's setting, this turns out to require that the likelihood ratio is increasing and concave. Kirkegaard (2014) proves that this type of justification of the FOA can be extended to many signals and many tasks, as long as the tasks are independent. In the case with two signals and two tasks, the appropriate assumption is that the antiderivative of $F\left(x_{1}, x_{2} \mid a_{1}, a_{2}\right)$ is weakly convex in $\left(a_{1}, a_{2}\right)$, as described in the next assumption. Kirkegaard (2014) terms this condition the cumulative lower orthant convexity condition (CLOCC).

Assumption A3' (CLOCC): $F\left(x_{1}, x_{2} \mid a_{1}, a_{2}\right)$ satisfies the cumulative lower orthant convexity condition $(C L O C C)$, i.e.

$$
\int_{\underline{x}_{1}}^{x_{1}} \int_{\underline{x}_{2}}^{x_{2}} G^{1}\left(y_{1} \mid a_{1}\right) G^{2}\left(y_{2} \mid a_{2}\right) d y_{2} d y_{1}=\int_{\underline{x}_{1}}^{x_{1}} G^{1}\left(y_{1} \mid a_{1}\right) d y_{1} \int_{\underline{x}_{2}}^{x_{2}} G^{2}\left(y_{2} \mid a_{2}\right) d y_{2}
$$

is weakly convex in $\left(a_{1}, a_{2}\right)$ for all $\left(x_{1}, x_{2}\right)$ and all $\left(a_{1}, a_{2}\right)$.

Unfortunately, it is not possible to sign the second derivative of $v\left(w\left(x_{1}\right), x_{2}\right)$ with respect to $x_{1}$ in general. The next subsection considers a special case where it can be done, and where CLOCC may serve to justify the FOA.

However, it is straightforward to sign the second derivative of $v\left(w\left(x_{1}\right), x_{2}\right)$ with respect to $x_{2}$. In fact, by assumption, the agent's utility is concave in $x_{2}$. This observation opens the door for a simpler relaxation of Assumption A3. In particular, I will exploit that $v\left(w\left(x_{1}\right), x_{2}\right)$ is increasing in $x_{1}$ and increasing and concave in $x_{2}$. Thus, a hybrid of LOCC and CLOCC is called for.

Assumption A3" (HOCC): $F\left(x_{1}, x_{2} \mid a_{1}, a_{2}\right)$ satisfies the hybrid orthant convex- 
ity condition $(\mathrm{HOCC})$, i.e.

$$
G^{1}\left(x_{1} \mid a_{1}\right) \int_{\underline{x}_{2}}^{x_{2}} G^{2}\left(y_{2} \mid a_{2}\right) d y_{2}
$$

is weakly convex in $\left(a_{1}, a_{2}\right)$ for all $\left(x_{1}, x_{2}\right)$ and all $\left(a_{1}, a_{2}\right)$.

Assumptions A3, A3', and A3" can be ordered according to how restrictive they are. Specifically, Assumption A3 (LOCC) implies A3" (HOCC), which in turn implies A3' (CLOCC). As was the case for Assumption A3, Assumptions A3' and A3" both imply that each term in (12) and (13), respectively, must be strictly convex in $a_{i}$ for interior $x_{i}$. As before, HOCC is satisfied if e.g. the two terms are log-convex in $a_{1}$ and $a_{2}$, respectively. Although HOCC is weaker than LOCC, it is easy to see that it remains the case that $E U\left(a_{1}, a_{2}\right)$ is strictly concave in $a_{2}$, or $E U_{22}\left(a_{1}, a_{2}\right)<0$, regardless of the contract. Formally, this can be established by using integration by parts twice and invoking the assumption that $v_{22}<0$. Likewise, as before, $E U\left(a_{1}, a_{2}\right)$ is concave in $a_{1}$ whenever the contract is monotonic. While the agent's expected utility is thus concave in each task, it remains to show that it is jointly concave in $\left(a_{1}, a_{2}\right)$.

As explained in Kirkegaard (2014), multi-signal justifications of the FOA that rely on LOCC or CLOCC also require one to sign certain cross-partial derivatives. For instance, recall that $v_{12}<0$ was invoked to prove Lemma 1. Given HOCC, it turns out to be sufficient to add the mild assumption that $v_{122} \geq 0$. In other words, the price of weakening Assumption A3 by replacing it with Assumption A3" is that $v_{122} \geq 0$ must be assumed. However, note that if $x_{2}$ is income, then $v_{122} \geq 0$ is implied by Assumption A5. In this case, then, there is no cost of replacing A3 by A3". More generally, in view of Assumption 5, $v_{122} \geq 0$ is satisfied if e.g. $v_{2}\left(w, x_{2}\right)$ is log-supermodular in $\left(w, x_{2}\right)$, i.e. if the agent's risk aversion with respect to the private rewards is decreasing with labor income.

Assumption A8 (Supermodular marginal utility): The agent's marginal utility of the private reward is supermodular. That is, $v_{2}\left(w, x_{2}\right)$ is supermodular in $\left(w, x_{2}\right)$, or $v_{122}\left(w, x_{2}\right) \geq 0$.

Theorem 2 proves that the FOA remains valid once LOCC is replaced by 
HOCC, provided that Assumption A8 is imposed as well.

Theorem 2 Assume any second-best action $\left(a_{1}, a_{2}\right)$ is interior. Then, given Assumptions A1,A2,A3", and A4-A8, the FOA is valid.

Proof. The argument that $w\left(x_{1}\right)$ is increasing in $x_{1}$ remains unchanged as Assumption A3 is replaced by A3". Using integration by parts repeatedly yields

$$
\begin{aligned}
E U\left(a_{1}, a_{2}\right)= & v\left(w\left(\bar{x}_{1}\right), \bar{x}_{2}\right)-v_{2}\left(w\left(\bar{x}_{1}\right), \bar{x}_{2}\right) \int G^{2}\left(x_{2} \mid a_{2}\right) d x_{2} \\
& +\int v_{22}\left(w\left(\bar{x}_{1}\right), x_{2}\right) \int_{\underline{x}_{2}}^{x_{2}} G^{2}\left(y_{2} \mid a_{2}\right) d y_{2} d x_{2} \\
& -\int v_{1}\left(w\left(x_{1}\right), \bar{x}_{2}\right) w^{\prime}\left(x_{1}\right) G^{1}\left(x_{1} \mid a_{1}\right) d x_{1} \\
& +\int v_{12}\left(w\left(x_{1}\right), \bar{x}_{2}\right) w^{\prime}\left(x_{1}\right)\left(\int G^{2}\left(x_{2} \mid a_{2}\right) d x_{2} G^{1}\left(x_{1} \mid a_{1}\right)\right) d x_{1} \\
& -\iint v_{122}\left(w\left(x_{1}\right), x_{2}\right) w^{\prime}\left(x_{1}\right)\left(\int_{\underline{x}_{2}}^{x_{2}} G^{2}\left(y_{2} \mid a_{2}\right) d y_{2} G^{1}\left(x_{1} \mid a_{1}\right)\right) d x_{2} d x_{1} \\
& -c\left(a_{1}, a_{2}\right) .
\end{aligned}
$$

By assumption, $v_{1}, v_{2}>0>v_{12}, v_{22}$ and $v_{112} \geq 0$. Thus, by Assumption A3", $E U\left(a_{1}, a_{2}\right)$ is the sum of functions that are concave in $\left(a_{1}, a_{2}\right)$. Hence, the agent's utility is concave. The theorem now follows by the same arguments that established Theorem 1.

\subsection{Multiplicative rewards}

A particularly tractable type of utility function is introduced and analyzed next. From a technical perspective, the convenient functional form makes it possible to further relax Assumption A3. More importantly, in economics terms it is significant that the added structure allows further insights into the properties of the optimal contract. These implications are pursued in the next section.

Assume that the reward function is multiplicative, or that

$$
v\left(w, x_{2}\right)=-m(w) n\left(x_{2}\right),
$$


where $m$ and $n$ are strictly negative functions that are strictly increasing and strictly concave on their domain. Note that Assumptions A5 and A9 are trivially satisfied, as is the part of Assumption A4 that pertains to $v\left(w, x_{2}\right)$. An obvious example that satisfies (14) is $m(w)=-e^{-r w}$ and $n\left(x_{2}\right)=-e^{-r x_{2}}$, for any $r>0$. Then, $v\left(w, x_{2}\right)=-e^{-r\left(w+x_{2}\right)}$. Here, $x_{2}$ can be interpreted as income, and the agent exhibits constant absolute risk aversion (CARA). The example in Section 2 fits (14) as well, with $n\left(x_{2}\right)$ taking the value -1 or 0 .

Letting $\omega$ summarize the contract $w\left(x_{1}\right), x_{1} \in\left[\underline{x}_{1}, \bar{x}_{1}\right]$, define

$$
\begin{aligned}
M\left(a_{1} \mid \omega\right) & =\int m\left(w\left(x_{1}\right)\right) g^{1}\left(x_{1} \mid a_{1}\right) d x_{1}<0 \\
N\left(a_{2}\right) & =-\int n\left(x_{2}\right) g^{2}\left(x_{2} \mid a_{2}\right) d x_{2}>0
\end{aligned}
$$

such that

$$
E U\left(a_{1}, a_{2}\right)=M\left(a_{1} \mid \omega\right) N\left(a_{2}\right)-c\left(a_{1}, a_{2}\right) .
$$

Assumptions A3, A3' and A3" all imply that the expectation of $n\left(x_{2}\right)$ is strictly concave in $a_{2}$. By Assumption A2, the expectation is also strictly increasing in $a_{2}$. Hence, $N\left(a_{2}\right)$ is positive, strictly decreasing, and strictly concave.

In this multiplicative case, (9) can be written in a much simpler form,

$$
\left(\lambda N\left(a_{2}\right)+\mu_{2} N^{\prime}\left(a_{2}\right)\right)+\left(\mu_{1} N\left(a_{2}\right)\right) l_{a_{1}}^{1}\left(x_{1} \mid a_{1}\right)=\frac{1}{m^{\prime}(w)},
$$

or, by renaming the terms in the parentheses,

$$
\widehat{\lambda}+\widehat{\mu} l_{a_{1}}^{1}\left(x_{1} \mid a_{1}\right)=\frac{1}{m^{\prime}(w)}
$$

precisely as in the usual model with no private rewards. Standard methods can now be used to prove that $\mu_{1}>0$ (or $\widehat{\mu}>0$ ). Specifically, given MLRP, the contract would be non-increasing if $\mu_{1} \leq 0$, thus implying that $E U_{1}<0$ in violation of the incentive-compatibility constraints. In other words, given only that $a_{1}>\underline{a}_{1}$, it must hold that $\mu_{1}>0$ such that the optimal contract is nondecreasing in $x_{1}$ regardless of which $\left(a_{1}, a_{2}\right)$ the principal seeks to implement. Note that as long as wages are interior, this argument applies to all $\left(a_{1}, a_{2}\right)$ pairs 
with $a_{1}>\underline{a}_{1}$, not only the pair that turns out to be optimal. Note also that it is not necessary to sign $\mu_{2}$. Thus, there is no need to consider the doubly-relaxed problem, and thus no need to invoke Lemma 3, which is the only place where the assumptions that $B_{2}\left(a_{1}, a_{2}\right) \leq 0$ and $c_{12}\left(a_{1}, a_{2}\right) \geq 0$ are utilized. Thus, in the multiplicative model, part of Assumptions A4 and A6 can be relaxed. As only the relaxed problem is considered, Assumption A7 is modified as well.

Assumption A6' (The Principal's Preferences): The principal is risk neutral, with expected utility $B\left(a_{1}, a_{2}\right)-E\left[w \mid a_{1}, a_{2}\right]$.

Assumption A7' (The relaxed problem): A solution to the relaxed probelm exists. Any solution involves only wages in the interior of the domain of $v\left(w, x_{2}\right)$.

Jewitt's (1988) proof that $m\left(w\left(x_{1}\right)\right)$ may be concave applies to the current setting as well. He proves that this property holds if $l_{a_{1}}^{1}\left(x_{1} \mid a_{1}\right)$ is increasing and concave and

$$
\frac{d}{d w}\left(\frac{-m^{\prime \prime}(w)}{m^{\prime}(w)^{3}}\right) \geq 0 .
$$

The latter condition is satisfied if $m(w)=-e^{-r w}, r>0$, as in the CARA example. Note that if $m\left(w\left(x_{1}\right)\right)$ is increasing and concave in $x_{1}$, then so is $v\left(w\left(x_{1}\right), x_{2}\right)$. It is for this reason that Assumption A3' will prove to be sufficient to justify the FOA. However, to use Jewitt's argument, it is evidently necessary to strengthen Assumptions A2 and parts of A4. Recall that $c_{12}\left(a_{1}, a_{2}\right) \geq 0$ is no longer required.

Assumption A2' (Concave likelihood-Ratio): The marginal distributions satisfy Assumption A2 (MLRP). Moreover, $l_{a_{1}}^{1}\left(x_{1} \mid a_{1}\right)$ is weakly concave in $x_{1}$ for all $x_{1} \in\left[\underline{x}_{1}, \bar{x}_{1}\right]$.

Assumption A4' (Multiplicative Rewards): The agent's Bernoulli utility is $-m(w) n\left(x_{2}\right)-c\left(a_{1}, a_{2}\right)$. Costs, $c\left(a_{1}, a_{2}\right)$, are strictly increasing and weakly convex in $\left(a_{1}, a_{2}\right)$. Moreover, $m$ and $n$ are strictly negative functions that are strictly increasing and strictly concave on their domain. Finally, $m$ satisfies (18).

The FOA can now be justified in the multiplicative model.

Theorem 3 Assume any second-best action $\left(a_{1}, a_{2}\right)$ is interior. Then, given Assumptions A1,A2',A3',A4', A6', and A7', the FOA is valid. 
Proof. Starting from the expression of $E U\left(a_{1}, a_{2}\right)$ derived in Theorem 2, another round of integration by parts leads to a new expression that depends only on the terms in Assumption A3' (rather than A3" as in Theorem 2). Concavity then obtains if the derivatives of $u\left(x_{1}, x_{2}\right)=v\left(w\left(x_{1}\right), x_{2}\right)$ have the correct sign. It is required that $u_{1}, u_{2} \geq 0 \geq u_{11}, u_{12}, u_{22}$ and $u_{112}, u_{122} \geq 0 \geq u_{1122}$. Assumptions A2 and A4 together implies that $u_{11} \leq 0$ (as $m\left(w\left(x_{1}\right)\right)$ is concave in $\left.x_{1}\right)$. Given the multiplicative nature of $u$, it then follows that the second set of inequalities is also satisfied. Thus, the agent's utility is concave.

\section{Intrinsic and extrinsic motivation}

The multiplicative model in Section 5.2 can be used to illustrate some key properties of the optimal contract. Incidentally, the current section does not require a thorough understanding of the preceding material on how to justify the FOA. ${ }^{15}$

Thus, assume that $v\left(w, x_{2}\right)$ takes the form in (14). The set of implementable actions is characterized first. To begin, fix some interior action that the principal might like to induce. Then, $M\left(a_{1} \mid \omega\right)$ and $M^{\prime}\left(a_{1} \mid \omega\right)$ are characterized completely by the local incentive compatibility constraints that $E U_{1}=E U_{2}=0$, with

$$
M^{\prime}\left(a_{1} \mid \omega\right)=\frac{c_{1}\left(a_{1}, a_{2}\right)}{N\left(a_{2}\right)}, M\left(a_{1} \mid \omega\right)=\frac{c_{2}\left(a_{1}, a_{2}\right)}{N^{\prime}\left(a_{2}\right)},
$$

where $M^{\prime}\left(a_{1} \mid \omega\right)$ denotes the derivative of $M\left(a_{1} \mid \omega\right)$ with respect to $a_{1}$, holding fixed the contract. An outside observer who does not realize that $a_{2}$ is endogenous might reasonably interpret $M\left(a_{1} \mid \omega\right)$ as measuring the agent's "base utility" at work and $M^{\prime}\left(a_{1} \mid \omega\right)$ as measuring the intensity of the explicit or extrinsic incentives. Of course, these are local measures as they are evaluated at the $a_{1}$ value induced by the contract $\omega$.

For ease of exposition, assume that for any interior action there exists a contract satisfying (19). Assume also that $c_{12}\left(a_{1}, a_{2}\right) \geq 0$.

Fixing an interior action, $N\left(a_{2}\right)$ and $c\left(a_{1}, a_{2}\right)$ are exogenous while $M\left(a_{1} \mid \omega\right)$

\footnotetext{
${ }^{15}$ However, it is important to recall $(i)$ the information structure and (ii) the basics of the multiplicative model. The former is described in the paragraph before Assumption A1 in Section 2. The latter is described in the first three paragraphs of Section 4.2.
} 
is determined by incentive compatibility. Thus, the agent's utility is already determined. Consequently, there may be actions for which the participation constraint is slack or redundant and others for which it is violated. Given (19), the participation constraint is satisfied if and only if

$$
\frac{c_{2}\left(a_{1}, a_{2}\right)}{N^{\prime}\left(a_{2}\right)} N\left(a_{2}\right)-c\left(a_{1}, a_{2}\right) \geq \bar{u}
$$

when $a_{2}$ is interior. Simple differentiation shows that the left hand side is strictly decreasing in $a_{2}$. Assuming (20) is satisfied at $\underline{a}_{2}$, define

$$
t\left(a_{1}\right)=\max \left\{a_{2} \in\left[\underline{a}_{2}, \bar{a}_{2}\right] \mid \frac{c_{2}\left(a_{1}, a_{2}\right)}{N^{\prime}\left(a_{2}\right)} N\left(a_{2}\right)-c\left(a_{1}, a_{2}\right) \geq \bar{u}\right\}
$$

as the threshold value of $a_{2}$ such that (20) holds for all $a_{2}$ below that value. Let $t\left(a_{1}\right)=\underline{a}_{2}$ if $(20)$ is violated at $\underline{a}_{2}$.

If $t\left(a_{1}\right)$ is interior, (20) is satisfied if and only if $a_{2} \in\left[\underline{a}_{2}, t\left(a_{1}\right)\right]$. In other words, only $a_{2}$ levels at or below the threshold $t\left(a_{1}\right)$ can be implemented. If $t\left(a_{1}\right)=\bar{a}_{2}$, no interior $a_{2}$ can be implemented at all. Finally, if $t\left(a_{1}\right)=\underline{a}_{2}$, then only $\underline{a}_{2}$ can be implemented. ${ }^{16}$ As this case is less interesting, it will be ignored in the remainder. Assuming then that $t\left(a_{1}\right)>\underline{a}_{2},(20)$ must be slack for any $a_{2} \in\left(\underline{a}_{2}, t\left(a_{1}\right)\right)$, implying that the agent earns more than reservation utility. However, the participation constraint binds at $a_{2}=t\left(a_{1}\right)$, even if $t\left(a_{1}\right)=\bar{a}_{2} \cdot{ }^{17}$

Since (20) is also strictly decreasing in $a_{1}, t\left(a_{1}\right)$ is decreasing in $a_{1}$, with $t^{\prime}\left(a_{1}\right)<0$ if $t\left(a_{1}\right)$ is interior. Hence, the larger $a_{1}$ is, the smaller is the set of implementable $a_{2}$ values. This completes the description of the feasible set of actions, illustrated as the shaded area in Figure 1. Note that the agent's expected utility is increasing towards the south-west. ${ }^{18}$ As explained in the next subsection, a key reason the current model delivers richer predictions than the

\footnotetext{
${ }^{16} \mathrm{At} \underline{a}_{2}$, the incentive compatibility constraint that $E U_{2} \leq 0$ is equivalent to $M\left(a_{1} \mid \omega\right) \geq$ $\frac{c_{2}\left(a_{1}, \underline{a}_{2}\right)}{N^{\prime}\left(\underline{a}_{2}\right)}$. Thus, even if (20) is violated at $\underline{a}_{2}, M\left(a_{1} \mid \omega\right)$ can be increased to induce participation without violating incentive compatibility. Hence, $\underline{a}_{2}$ is implementable as long as $c_{2}\left(a_{1}, \underline{a}_{2}\right)>0$.

${ }^{17}$ At $\bar{a}_{2}$, the incentive compatibility constraint that $E U_{2} \geq 0$ is equivalent to $M\left(a_{1} \mid \omega\right) \leq$ $\frac{c_{2}\left(a_{1}, \underline{a}_{2}\right)}{N^{\prime}\left(\underline{a}_{2}\right)}$. Thus, $M\left(a_{1} \mid \omega\right)$ can be lowered to make the participation constraint bind.

${ }^{18}$ Recall that the contract must change in order to implement a different action. Thus, the contracts are not the same across different points in the figure.
} 


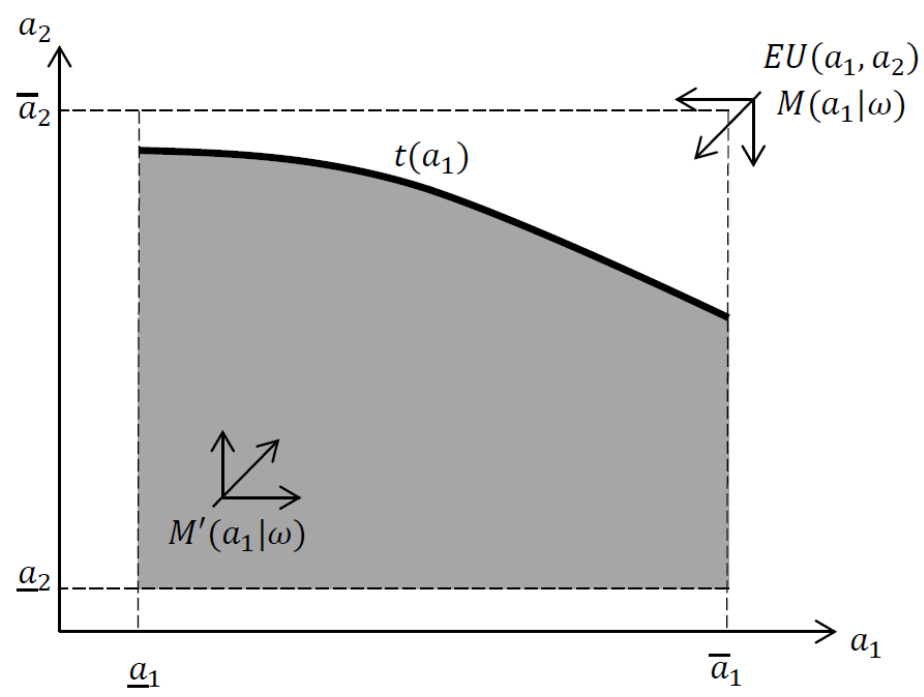

Figure 1: The feasible set and some comparative statics.

LEN model is that the feasible set in the latter is simply a curve. That is, for each $a_{1}$ there is a unique implementable $a_{2}$ value in the LEN model.

It is interesting to analyze how the properties of the contract depends on which action in the feasible set is implemented. For brevity, I examine interior actions only. Consider first how the contract depends on $a_{2}$. Keeping (19) in mind, note that

$$
\begin{aligned}
& \frac{\partial}{\partial a_{2}}\left(\frac{c_{1}\left(a_{1}, a_{2}\right)}{N\left(a_{2}\right)}\right)=\frac{c_{12}\left(a_{1}, a_{2}\right) N\left(a_{2}\right)-c_{1}\left(a_{1}, a_{2}\right) N^{\prime}\left(a_{2}\right)}{N\left(a_{2}\right)^{2}}>0 \\
& \frac{\partial}{\partial a_{2}}\left(\frac{c_{2}\left(a_{1}, a_{2}\right)}{N^{\prime}\left(a_{2}\right)}\right)=\frac{c_{22}\left(a_{1}, a_{2}\right) N^{\prime}\left(a_{2}\right)-c_{2}\left(a_{1}, a_{2}\right) N^{\prime \prime}\left(a_{2}\right)}{N^{\prime}\left(a_{2}\right)^{2}}<0
\end{aligned}
$$

since $N\left(a_{2}\right), N^{\prime \prime}\left(a_{2}\right)>0>N^{\prime}\left(a_{2}\right)$. Holding $a_{1}$ fixed, it follows that $M\left(a_{1} \mid \omega\right)$ and $M^{\prime}\left(a_{1} \mid \omega\right)$ are inversely related, as described in the introduction. That is, let $\omega^{\prime}$ and $\omega^{\prime \prime}$ denote two contracts that induce the same $a_{1}$ but different $a_{2}$ like $a_{2}^{\prime}$ and $a_{2}^{\prime \prime}$, respectively, with $a_{2}^{\prime \prime}>a_{2}^{\prime}$. Then, $M\left(a_{1} \mid \omega^{\prime}\right)>M\left(a_{1} \mid \omega^{\prime \prime}\right)$ and $M^{\prime}\left(a_{1} \mid \omega^{\prime}\right)<M^{\prime}\left(a_{1} \mid \omega^{\prime \prime}\right)$. The aforementioned outsider would thus conclude that $\omega^{\prime}$ delivers higher "base utility" than $\omega^{\prime \prime}$ but weaker explicit incentives. Nevertheless, the agent works equally hard on the job with either contract. Intuitively, higher "base utility" at work makes private rewards less important, thus lessening the 
agent's incentives to pursue such rewards. Labor income then contributes more significantly to the agent's overall utility, which is why weaker explicit incentives are required to induce the same effort on the job.

Consider next the contract's dependence upon $a_{1}$. Here,

$$
\begin{aligned}
& \frac{\partial}{\partial a_{1}}\left(\frac{c_{1}\left(a_{1}, a_{2}\right)}{N\left(a_{2}\right)}\right)=\frac{c_{11}\left(a_{1}, a_{2}\right)}{N\left(a_{2}\right)} \geq 0 \\
& \frac{\partial}{\partial a_{1}}\left(\frac{c_{2}\left(a_{1}, a_{2}\right)}{N^{\prime}\left(a_{2}\right)}\right)=\frac{c_{12}\left(a_{1}, a_{2}\right)}{N^{\prime}\left(a_{2}\right)} \leq 0 .
\end{aligned}
$$

Holding $a_{2}$ fixed but varying $a_{1}$, it is once again the case that the agent's base utility from work is inversely related to the steepness of the incentives. Let $\omega^{\prime}$ and $\omega^{\prime \prime}$ denote two contracts that induce a fixed $a_{2}$ but different $a_{1}$ such as $a_{1}^{\prime}$ and $a_{1}^{\prime \prime}$, respectively, with $a_{1}^{\prime \prime}>a_{1}^{\prime}$. Then, $M\left(a_{1}^{\prime} \mid \omega^{\prime}\right) \geq M\left(a_{1}^{\prime \prime} \mid \omega^{\prime \prime}\right)$ and $M^{\prime}\left(a_{1}^{\prime} \mid \omega^{\prime}\right) \leq M^{\prime}\left(a_{1}^{\prime \prime} \mid \omega^{\prime \prime}\right)$. The latter is intuitive; steeper incentives are required to make the agent work harder on the job, other things equal. The first property is perhaps more surprising at first blush; the agent's reward at work is lower when he is induced to work harder. Contrary to the standard model, however, the agent's utility is not pegged down by the participation constraint. Instead, it is determined by incentive compatibility. When $a_{1}$ increases, the marginal cost of the private task, $c_{2}$, increases as well. To maintain unchanged incentives to pursue private rewards, these must be made more important in the agent's utility. Thus, the reward from work must decrease.

In summary, $M\left(a_{1} \mid \omega\right)$ is decreasing in both $a_{1}$ and $a_{2}$ while $M^{\prime}\left(a_{1} \mid \omega\right)$ is increasing in both (recall that $\omega$ changes with the action to be implemented). See Figure 1. The costs of inducing different feasible actions is studied next.

Fix a feasible $\left(a_{1}, a_{2}\right)$ pair, and let $C\left(a_{1}, a_{2}\right)$ denote the implementation costs. Let $C\left(a_{1}, \underline{a}_{2}\right)=\infty$ if $\underline{a}_{2}$ is not implementable, i.e. if $c_{2}\left(a_{1}, \underline{a}_{2}\right)=0$. If $\underline{a}_{2}$ is implementable, the incentive compatibility constraint that $E U_{2} \leq 0$ reduces to $M\left(a_{1}\right) \geq \frac{c_{2}\left(a_{1}, \underline{a}_{2}\right)}{N^{\prime}\left(\underline{a}_{2}\right)}$. If the inequality is strict, the principal can reduce $m\left(w\left(x_{1}\right)\right)$ by the same amount in every state without affecting the $E U_{1}=0$ constraint. Since this would reduce implementation costs, the conclusion must be that the optimal contract satisfies $E U_{2}=0$. From (20), the participation constraint is slack. Stated differently, as long as $a_{1}$ is interior and $a_{2} \in\left[\underline{a}_{2}, t\left(a_{1}\right)\right)$, the relevant 
constraints are that $E U_{1}=0$ and $E U_{2}=0$. It can now be shown that $C\left(a_{1}, a_{2}\right)$ is decreasing in $a_{2}$ on $\left[\underline{a}_{2}, t\left(a_{1}\right)\right]$ if

$$
\frac{\partial}{\partial a_{2}}\left(\frac{c_{2}\left(a_{1}, a_{2}\right)}{c_{1}\left(a_{1}, a_{2}\right)} \frac{N\left(a_{2}\right)}{N^{\prime}\left(a_{2}\right)}\right) \leq 0
$$

for all $\left(a_{1}, a_{2}\right)$. This is a relatively mild assumption. It is satisfied if $c_{12}$ is small and $N\left(a_{2}\right)$ is log-convex, the latter of which is ensured if $G^{2}\left(x_{2} \mid a_{2}\right)$ or its antiderivative (the counterpart to (11)) is log-convex in $a_{2}$. Recall that Assumptions A3, A3', and A3" are satisfied if both $G^{1}$ and $G^{2}$ have that property.

Proposition 1 Assume utility from rewards are multiplicative, (21) holds, and that the assumptions in one of Theorems 1, 2, or 3 hold. Assume wages are interior regardless of which feasible $\left(a_{1}, a_{2}\right)$ pair the principal seeks to implement. Then, for any $a_{1} \in\left(\underline{a}_{1}, \bar{a}_{1}\right)$ for which $t\left(a_{1}\right)>\underline{a}_{2}, C\left(a_{1}, a_{2}\right)$ is strictly decreasing in $a_{2}$ on $\left[\underline{a}_{2}, t\left(a_{1}\right)\right]$.

Proof. In the Appendix.

As before, let $B\left(a_{1}, a_{2}\right)$ denote the principal's direct utility from the action. Assume, for now, that $B_{2}=0$. Proposition 1 then signifies that for any (interior) $a_{1}$, the optimal $a_{2}$ to induce is on the boundary of the feasible set, $a_{2}=t\left(a_{1}\right)$. The same conclusion of course holds if $B_{2} \geq 0$. Consequently, the participation constraint is binding regardless of which $a_{1}$ the principal seeks to implement. Since $t\left(a_{1}\right)$ is decreasing it follows that if the principal desires the agent to work harder on the job, then it is optimal to simultaneously induce the agent to work less hard in the pursuit of private rewards.

Given $a_{1}$ is to be implemented, the agent's total costs is thus $c\left(a_{1}, t\left(a_{1}\right)\right)$. To proceed, assume that $t\left(a_{1}\right)$ is interior. Although $a_{1}$ and $t\left(a_{1}\right)$ move in opposite directions, (21) implies that

$$
\frac{\partial c\left(a_{1}, t\left(a_{1}\right)\right)}{\partial a_{1}} \geq 0 .
$$

That is, the agent's total cost of effort increases when he is induced to work harder, even though he works less intensively on the other task. ${ }^{19}$

\footnotetext{
${ }^{19}$ From $(21), t^{\prime}\left(a_{1}\right) \geq-\frac{c_{1}\left(a_{1}, t\left(a_{1}\right)\right)}{c_{2}\left(a_{1}, t\left(a_{1}\right)\right)}$ which in turn yields $(22)$.
} 
Compare two $a_{1}$ levels, $a_{1}^{\prime \prime}$ and $a_{1}^{\prime}$, with $a_{1}^{\prime \prime}>a_{1}^{\prime}$. Let $\underline{\omega}^{\prime \prime}$ and $\underline{\omega}^{\prime}$ denote the contract that implements $a_{1}^{\prime \prime}$ and $a_{1}^{\prime}$, respectively, as cheaply as possible. Since the participation constraint binds in either case, but costs are higher when $a_{1}^{\prime \prime}$ is implemented, it follows that utility from both types of rewards must be greater under $a_{1}^{\prime \prime}$ than under $a_{1}^{\prime}$. That is,

$$
M\left(a_{1}^{\prime \prime} \mid \underline{\omega}^{\prime \prime}\right) N\left(t\left(a_{1}^{\prime \prime}\right)\right) \geq M\left(a_{1}^{\prime} \mid \underline{\omega}^{\prime}\right) N\left(t\left(a_{1}^{\prime}\right)\right) .
$$

However, since $t\left(a_{1}^{\prime \prime}\right)<t\left(a_{1}^{\prime}\right)$, the agent's private returns are worth less when $a_{1}=a_{1}^{\prime \prime}$. Hence, he must be compensated with higher rewards at work. Thus, perhpas unsurprisingly, $M\left(a_{1}^{\prime \prime} \mid \underline{\omega}^{\prime \prime}\right)>M\left(a_{1}^{\prime} \mid \underline{\omega}^{\prime}\right)$. Now, to induce an interior $a_{1}$ along with $a_{2}=t\left(a_{1}\right)$ it is necessary that $E U_{1}=0$, or

$$
M^{\prime}\left(a_{1} \mid \underline{\omega}\right)=\frac{c_{1}\left(a_{1}, t\left(a_{1}\right)\right)}{N\left(t\left(a_{1}\right)\right)} .
$$

To illustrate the main point in the simplest possible way, assume that marginal costs, $c_{1}\left(a_{1}, a_{2}\right)$, are constant. Then,

$$
M^{\prime}\left(a_{1}^{\prime \prime} \mid \underline{\omega}^{\prime \prime}\right)=\frac{c_{1}}{N\left(t\left(a_{1}^{\prime \prime}\right)\right)}<\frac{c_{1}}{N\left(t\left(a_{1}^{\prime}\right)\right)}=M^{\prime}\left(a_{1}^{\prime} \mid \underline{\omega}^{\prime}\right) .
$$

Hence, an outsider who fails to take into account that $a_{2}$ is endogenous would conclude that the marginal return to extra effort is lower the harder the agent is induced to work. ${ }^{20}$ In other words, it looks as if the agent works harder when given weaker explicit incentives. Corollary 1 summarizes.

Corollary 1 Assume (21) holds and that $c_{1}\left(a_{1}, a_{2}\right)$ is constant. Let $\underline{\omega}^{\prime \prime}$ and $\underline{\omega}^{\prime}$ denote the cost-minimizing contract that implements $a_{1}^{\prime \prime}$ and $a_{1}^{\prime}$, respectively, with $a_{1}^{\prime \prime}>a_{1}^{\prime}$. Assume $a_{1}$ and $t\left(a_{1}\right)$ are interior for $a_{1}=a_{1}^{\prime}, a_{1}^{\prime \prime}$. Then, $M\left(a_{1}^{\prime \prime} \mid \underline{\omega}^{\prime \prime}\right)>$ $M\left(a_{1}^{\prime} \mid \underline{\omega}^{\prime}\right)$ and $M^{\prime}\left(a_{1}^{\prime \prime} \mid \underline{\omega}^{\prime \prime}\right)<M^{\prime}\left(a_{1}^{\prime} \mid \underline{\omega}^{\prime}\right)$.

Consider now the possibility that the principal cares directly about $a_{2}$ as well, with $B_{2}<0$. Since cost minimization is then no longer the only concern relevant

\footnotetext{
${ }^{20}$ However, if $c_{1}$ is sufficiently steep, the conclusion that $M^{\prime}\left(a_{1}^{\prime \prime} \mid \underline{\omega}^{\prime \prime}\right)>M^{\prime}\left(a_{1}^{\prime} \mid \underline{\omega}^{\prime}\right)$ is obtained.
} 
to which $a_{2}$ to induce, the second-best action may now move into the interior of the feasible set. ${ }^{21}$ Hence, the participation constraint may be slack.

Corollary 2 The agent may earn economic rents if $B_{2}<0$.

The agent accrues rent due to what can be thought of as "incomplete" contracting as $x_{2}$ is non-verifiable. Although Corollary 2 might seem less surprising in this light, it is interesting to note that the participation constraint is always binding in the popular LEN model, even when allowing for private rewards. The next subsection contrasts the model in the current paper with the LEN model.

There are many situations in which the non-contractible uncertainty is payoff relevant to both the agent and the principal, as assumed in Corollary 2. For example, Holmström and Milgrom (1991) point out that a "contractor's performance (such as courtesy, attention to detail, or helpful advice) are unmeasurable but are enhanced by attention [...] spent on that activity". ${ }^{22}$ Another example they give is when one wants to motivate "teachers to teach both basic skills and higher-order thinking skills, but [...] higher-order thinking skills cannot be measured". Likewise, consider a salesman who represents several companies and who invests effort $a_{i}$ into understanding firm $i$ 's product line. The quantity of firm $i$ 's products that he manages to sell depends not only on $a_{i}$ but also on $a_{j}$, i.e. on how well he presents competing products. ${ }^{23}$ A similar story might hold for real estate agents.

\subsection{Comparison with the LEN model}

The Linear-Exponential-Normal model is due to Holmström and Milgrom (1987, 1991). The agent is assumed to exhibit constant absolute risk aversion. Importantly, costs are assumed to be monetary. Thus, the agent's Bernoulli utility is

\footnotetext{
${ }^{21}$ Note that as long as $t\left(a_{1}\right)$ is interior, wage costs are continuous on $\left[\underline{a}_{2}, t\left(a_{1}\right)\right]$ when $\underline{a}_{2}$ is implementable (and on $\left(\underline{a}_{2}, t\left(a_{1}\right)\right]$ otherwise); see the proof of Proposition 1. Then, the principal would prefer to induce some $a_{2}<t\left(a_{1}\right)$ if $B_{2}\left(a_{1}, t\left(a_{1}\right)\right)$ is sufficiently small (i.e. negative).

${ }^{22}$ Giving helpful advice may be time-consuming and thus carry with it the opportunity cost of giving up time that could be spent pursuing private rewards.

${ }^{23}$ Although firm $i$ 's signal (the volume of sales) depends on $a_{j}$, the model does accommodate some such settings as described in footnote 7 . For instance, let $t_{i}\left(a_{1}, a_{2}\right)=2 a_{i}-a_{j}$ be a parameter in the distribution of $x_{1}$, with $a_{1}, a_{2} \in \mathbb{R}$. Thinking of $t_{1}$ and $t_{2}$ as the choice variables (such that $\left.a_{i}=\left(2 t_{i}+t_{j}\right) / 3\right)$, the cost function is $C\left(t_{1}, t_{2}\right)=c\left(\left(2 t_{1}+t_{2}\right) / 3,\left(2 t_{2}+t_{1}\right) / 3\right)$. It can be verified that $C\left(t_{1}, t_{2}\right)$ is convex if $c\left(a_{1}, a_{2}\right)$ is convex.
} 
$u\left(w, a_{1}, a_{2}\right)=-e^{-r\left(w-c\left(a_{1}, a_{2}\right)\right)}$ when he is paid $w$ and his action is $\left(a_{1}, a_{2}\right)$. Signals are assumed to be jointly normally distributed. In one interpretation of the model, the agent's action reduces to picking the means of these signals (the covariance matrix is beyond control). Finally, contracts are restricted to be linear in the signals. Holmström and Milgrom (1987) develop a dynamic micro-foundation for this static model which applies in some cases.

Assume there are two signals, $x_{1}$ and $x_{2}$. Given a contract $w\left(x_{1}, x_{2}\right)=\beta+$ $\alpha_{1} x_{1}+\alpha_{2} x_{2}$, the agent's certainty equivalent can be shown to equal

$$
C E\left(a_{1}, a_{2} \mid \beta, \alpha_{1}, \alpha_{2}\right)=\beta+\alpha_{1} a_{1}+\alpha_{2} a_{2}-c\left(a_{1}, a_{2}\right)+k\left(\alpha_{1}, \alpha_{2}\right),
$$

where $a_{i}$ is the mean of $x_{i}$, which is controlled by the agent, and where $k\left(\alpha_{1}, \alpha_{2}\right)$ depends on the terms in the covariance matrix but not on $a_{1}$ and $a_{2}$. Private rewards that are monetary and stochastic can be incorporated by letting $x_{2}$ be non-contractible and letting $\alpha_{2}>0$ be an exogenous constant that measures how rewarding the activity is. On the other hand, deterministic but non-monetary private rewards can be modelled by fixing $\alpha_{2}=0$ and assuming that $c\left(a_{1}, a_{2}\right)$ is $\mathrm{U}$ shaped in $a_{2}$. Holmström and Milgrom (1991) utilize the latter model. However, given the additive nature of the certainty equivalent in the LEN model, the two models are essentially isomorphic as one may simply think of $C\left(a_{1}, a_{2}\right)=\alpha_{2} a_{2}-$ $c\left(a_{1}, a_{2}\right)$ as a cost function that incorporates private rewards. Thus, assume from now on that $\alpha_{2}$ is a non-negative exogenous constant (possibly zero). Assume that $c$ is weakly convex such that the first order conditions are sufficient to identify an interior maximum of the $C E$. Assume that $c_{2}=\alpha_{2}$ somewhere in the interior, and that $c_{12}>0$. The case where $c_{12}<0$ yields analogous results.

Clearly, $\beta$ does not influence incentives at all, beyond participation. One conceptual advantage of the LEN model is that there is a simple measure of the strength of incentives, namely $\alpha_{1}$, whereas I had to resort to using $M^{\prime}\left(a_{1} \mid \omega\right)$ in the multiplicative model. On the other hand, the principal has only one instrument at his disposal when attempting to induce some $\left(a_{1}, a_{2}\right)$ pair in the LEN model.

To complete the analysis of the LEN model, assume for simplicity that $c_{11} c_{22}-$ $c_{12}^{2}>0$ and $c_{12}>0 .{ }^{24}$ Then, there is a unique $\left(a_{1}, a_{2}\right)$ pair which satisfies the

\footnotetext{
${ }^{24}$ Holmström and Milgrom (1991, Section 3) consider a version of the model where the cost
} 
first order conditions for any fixed $\left(\alpha_{1}, \alpha_{2}\right)$. Moreover, $a_{1}$ is strictly increasing in $\alpha_{1}$, while $a_{2}$ is strictly decreasing in $\alpha_{1} \cdot{ }^{25}$ Two important conclusions follow:

1. for any $a_{1}$ the principal might want to induce, there is exactly one implementable $a_{2}$ value, and

2. there is a unique $\alpha_{1}$ coefficient that implements that particular $a_{1}$ value (and its $a_{2}$ companion).

The first property does not generally hold in the current paper. This is of course particularly significant when the principal has preferences over $a_{2}$ as well. In this case, the agent may earn economic rent in the multiplicative model, but never in the LEN model where $\beta$ is adjusted to absorb all the rent. Note also that in the LEN model, $a_{1}$ and $a_{2}$ are competing in the sense that they must necessarily move in opposite directions. In contrast, the multiplicative model signifies that there are environments in which the principal has greater freedom to influence all dimensions of the agent's action. In particular, it is conceivable that a change in $B\left(a_{1}, a_{2}\right)$ will lead the principal to induce the agent to work less hard on both tasks, something that is impossible in the LEN model. In fact, combining the two properties of the LEN model reveals that for any desired $a_{1}$, there is a unique (linear) contract that can be rationalized $-\alpha_{1}$ is determined by incentive compatibility, $\beta$ by the participation constraint. In the multiplicative model, however, the optimal contract that implements a given $a_{1}$ depends on the principal's preferences over $a_{2}$.

The second property in particular is pertinent to the discussion of intrinsic and extrinsic motivation. Specifically, the LEN model can not reproduce the result that the same $a_{1}$ can be implemented with different contracts that vary in the strength of extrinsic incentives, at least when these are measured by the coefficient $\alpha_{1}$. An important insight of the multiplicative model is that the agent's effort on the job is generally not only determined by the steepness of the contract; there is an offsetting level effect.

function takes the form $c\left(a_{1}+a_{2}\right)$, in which case $c_{11} c_{22}-c_{12}^{2}=0$. The two main properties of the LEN model identified below also hold with this specification.

${ }^{25}$ An exception (which violates the above assumptions) occurs when $c\left(a_{1}, a_{2}\right)=c\left(a_{1}\right)+\gamma a_{2}$. Here, the agent's choice of $a_{2}$ is independent of the contract whenever $\gamma \neq \alpha_{2}$. If $\gamma=\alpha_{2}$, the agent is indifferent between all values of $a_{2}$. 


\section{Discussion}

In the standard single-task model, $\mu_{1}>0$ implies that the principal is better off if the agent (by mistake) works marginally higher than $a_{1}^{*}$ on the job. The same is true with private rewards, though it takes slightly more effort to establish it.

Proposition 2 Assume the second-best action is in the interior. Then, given Assumptions A1-A8, the principal is (weakly) better off if the agent works marginally harder than $a_{1}^{*}$, or

$$
B_{1}\left(a_{1}^{*}, a_{2}^{*}\right)-\int w\left(x_{1}\right) g_{a_{1}}^{1}\left(x_{1} \mid a_{1}^{*}\right) d x_{1} \geq 0
$$

Proof. Given $E U_{1}\left(a_{1}^{*}, a_{2}^{*}\right)=0$, the adjoint equation for $a_{1}$ reduces to

$$
B_{1}\left(a_{1}^{*}, a_{2}^{*}\right)-\int w\left(x_{1}\right) g_{a_{1}}^{1}\left(x_{1} \mid a_{1}^{*}\right) d x_{1}+\mu_{1} E U_{11}\left(a_{1}^{*}, a_{2}^{*}\right)+\mu_{2} E U_{12}\left(a_{1}^{*}, a_{2}^{*}\right)=0
$$

Now, substitute (10) into (25), to get

$$
\left(B_{1}\left(a_{1}^{*}, a_{2}^{*}\right)-\int w\left(x_{1}\right) g_{a_{1}}^{1}\left(x_{1} \mid a_{1}^{*}\right) d x_{1}\right)+\mu_{1} \frac{E U_{11}\left(a_{1}^{*}, a_{2}^{*}\right) E U_{22}\left(a_{1}^{*}, a_{2}^{*}\right)-E U_{12}\left(a_{1}^{*}, a_{2}^{*}\right)^{2}}{E U_{22}\left(a_{1}^{*}, a_{2}^{*}\right)}=0 .
$$

Recall that $E U_{22}\left(a_{1}^{*}, a_{2}^{*}\right)<0$. Since $E U\left(a_{1}, a_{2}\right)$ is concave the numerator must be non-negative. Then, (24) follows from $\mu_{1}>0$.

Since the optimal contract is monotonic, the last term on the left hand side in (24) is strictly positive; wage costs are higher when the agent works harder on task $a_{1}$. Hence, $(24)$ necessitates that $B_{1}\left(a_{1}^{*}, a_{2}^{*}\right)>0$. That is, $\left(a_{1}^{*}, a_{2}^{*}\right)$ can be optimal only if the principal's benefit function is increasing in $a_{1}$ at that point.

Although it is intuitive that $\mu_{2}<0$, it is instructive to consider a thought experiment where $x_{2}$ can be contracted upon. For simplicity, think of $x_{2}$ as outside income. Then, the principal can simply appropriate the private rewards. This is now a more or less standard moral hazard problem, in which case Kirkegaard (2014) shows that both multipliers are strictly positive. Hence, with private rewards, the negative multiplier is not due to the outside rewards as such, but rather due entirely to the assumption that the rewards are secret. Recall that a similar observation explains why the participation constraint may be slack. 


\section{Conclusion}

The current paper extends the canonical principal-agent model to allow the agent to pursue private, stochastic, and possibly non-monetary rewards.

From a technical point of view, a justification of the first-order approach (FOA) in this setting necessitates an understanding of the basic moral hazard problem with multi-tasking. However, multi-tasking has been largely ignored in the literature until very recently (the LEN model being an exception). The justification of the FOA presented here thus builds upon Kirkegaard's (2014) analysis. As explained there, the main technical cost of allowing multi-tasking is that outcomes from different tasks must be stochastically independent. With this restriction in place, however, the current paper establishes additional conditions under which Kirkegaard's (2014) justifications extends to private rewards. Once the required assumptions on the technology have been made to handle multi-tasking, as identified in Kirkegaard (2014), the economically significant assumptions are that the agent perceives outcomes and tasks to be substitutes and that his absolute risk aversion over labor income is decreasing in the private reward. It should be stressed that these assumptions appear to be rather mild. In other words, the costs of permitting private rewards are low.

The model of private rewards presented here is fairly simple, and thus abstracts away from a few potentially important complications. As just mentioned, a key assumption is that rewards are independent. However, it is not inconceivable that for example the gifts parents bestow on their children depend on the job held by the latter. Strictly speaking, the model does not allow the distribution of private rewards to be a direct function of the contract. However, the distribution could depend on the type of profession the agent is employed in, much in the same way that the outside option is likely to be a function of the agent's profession or level of education.

Although there are thus several directions in which the technical results could conceivably be extended in future research, the current model already identifies important economic insights. Conceptually, "unpacking" leisure by recognizing that rewards earned while not on the job are also endogenous reveals that the principal manipulates the agent's "work-life balance" through his contract design. 
Low base utility at work may entice the agent to focus more on pursuing private rewards and so steeper incentives on the job are required to compete for the agent's attention. Conversely, higher base utility at work reduces the incentive to pursue outside rewards. To an outside observer, the agent may now appear more "intrinsically motivated" as he can be induced to work hard on the job with flatter extrinsic incentives. In this respect, the model contributes to the behavioral contract theory literature by offering another perspective on intrinsic versus extrinsic motivation.

Space limitations prevent a fuller exploration of the model's potential to shed light on other economic phenomena. One question left for future research is whether differences in the emphasis placed on private rewards by observably different groups, like men and women, may explain why such groups are sometimes compensated in different ways despite only slight, if any, differences in performance. Having justified the FOA provides a method for researchers to explore this and other questions in the future. The multiplicative model in particular is quite tractable, yet richer than the LEN model. Finally, understanding contracting under private rewards represents a necessary first step in a longer-horizon endeavour to analyze common agency under various informational assumptions. Kirkegaard (2015) explores some first implications of the model in this regard.

\section{References}

Ábrahám, Á., Koehne, S. and N. Pavoni (2011): "On the first-order approach in principal-agent models with hidden borrowing and lending," Journal of Economic Theory, 146: 1331-1361.

Athey, S. (2002): "Monotone Comparative Statics under Uncertainty," Quarterly Journal of Economics, 117: 187-223.

Bénabou, R. and J. Tirole (2003): "Intrinsic and Extrinsic Motivation," Review of Economic Studies, 70: 289-520.

Conlon, J.R. (2009): "Two new Conditions Supporting the First-Order Approach to Multisignal Principal-Agent Problems," Econometrica, 77 (1): 249-278. 
Englmaier, F. and S. Leider (2012): "Contractual and Organizational Structure with Reciprocal Agents," American Economic Journal: Microeconomics, 4 (2): 146-183.

Holmström, B. and P. Milgrom (1987): "Aggregation and Linearity in the Provision of Intertemporal Incentives," Econometrica, 55 (2): 303-328.

Holmström, B. and P. Milgrom (1991): "Multitask Principal-Agent Analyses: Incentive Contracts, Asset Ownership, and Job Design," Journal of Law, Economics, and Organization, 24-52.

Jewitt, I. (1988): "Justifying the First-Order Approach to Principal-Agent Problems," Econometrica, 56 (5): 1177-1190.

Laffont, J-J. and D. Martimort (2002): The Theory of Incentives: The PrincipalAgent Model, Princeton University Press.

Ligon, J.A. and P.D. Thistle (2013): "Background Risk in the Principal-Agent Model," The Geneva Risk and Insurance Review, 38: 115-126.

Kirkegaard, R. (2014): "A Unifying Approach to Incentive Compatibility in Moral Hazard Problems", mimeo (October 2014).

Kirkegaard, R. (2015): "Common Agency with Disjoint Information," mimeo (April 2015).

Köszegi, B. (2014): "Behavioral Contract Theory," Journal of Economic Literature, 52 (4): 1075-1118.

Rogerson, W.P. (1985): "The First-Order Approach to Principal-Agent Problems," Econometrica, 53 (6): 1357-1367.

\section{Appendix}

Proof of Proposition 1. Fix an implementable $\left(a_{1}, a_{2}\right)$ pair where the participation constraint is slack, or $a_{2} \in\left[\underline{a}_{2}, t\left(a_{1}\right)\right)$. Formulate the cost-minimization 
problem that derives the cheapest contract that induces $\left(a_{1}, a_{2}\right)$ subject only to the incentive compatibility constraints that $E U_{1}=E U_{2}=0$, i.e.

$$
\begin{gathered}
\max _{w}\left(-\int w\left(x_{1}\right) g_{1}\left(x_{1} \mid a_{1}\right) d x_{1}\right) \\
\text { st. } \quad \operatorname{EU}_{i}\left(a_{1}, a_{2}\right)=0, i=1,2 .
\end{gathered}
$$

By assumption, the optimal contract involves only interior wages. As established in Section 5.2, the contract is monotonic and the multiplier to the first constraint is strictly positive, $\mu_{1}>0$, even if $\left(a_{1}, a_{2}\right)$ is not a second-best action (see the paragraph before Assumption A6'). Given the assumptions in any of Theorems 1-3, the agent's expected utility is thus concave. Thus, the local incentive compatibility constraints are sufficient. Stated differently, in the multiplicative model the above problem correctly identifies the cheapest contract that implements $\left(a_{1}, a_{2}\right)$, even if $\left(a_{1}, a_{2}\right)$ is not second-best. Hence, (26) identifies $-C\left(a_{1}, a_{2}\right)$.

The Envelope Theorem implies that a marginal change in $a_{2}$ causes $-C\left(a_{1}, a_{2}\right)$ to change by $\mu_{1} E U_{12}+\mu_{2} E U_{22}$, where $E U_{12}, E U_{22}<0$ and $\mu_{1}>0>\mu_{2} \cdot{ }^{26}$ Thus, wage costs decrease if $\mu_{1}$ is small compared to $\mu_{2}$. Intuitively, since $a_{1}$ and $a_{2}$ are substitutes, an increase in $a_{2}$ makes it harder to satisfy the incentive compatibility constraint that $E U_{1} \geq 0$. On the other hand, the constraint that $E U_{2} \leq 0$ is easier to satisfy, since the agent's problem is concave in $a_{2}$.

Next, Jewitt's (1988) famously elegant proof that $\mu_{1} c_{1}>0$ in the standard single-task model is modified to establish that

$$
\mu_{1} c_{1}\left(a_{1}, a_{2}\right)+\mu_{2} c_{2}\left(a_{1}, a_{2}\right)<0
$$

in the multiplicative model, whenever the participation constraint is slack. This inequality bounds $\mu_{1}$ relative to $\mu_{2}$. Since $E U_{1}=0, M^{\prime}\left(a_{1}\right) N\left(a_{2}\right)-c_{1}\left(a_{1}, a_{2}\right)=0$ or

$$
\int m\left(w\left(x_{1}\right)\right) \mu_{1} l_{a_{1}}^{1}\left(x_{1} \mid a_{1}\right) g^{1}\left(x_{1} \mid a_{1}\right) d x_{1} N\left(a_{2}\right)=\mu_{1} c_{1}\left(a_{1}, a_{2}\right)
$$

Borrowing a trick from Jewitt (1988), solve (17) for $\mu_{1} l_{a_{1}}^{1}$, keeping in mind that

\footnotetext{
${ }^{26}$ As mentioned, $\mu_{1}>0$. Since the participation constraint is slack and thus ignored, $\lambda=0$. Then, (17) would be violated for some $x_{1}$ unless $\mu_{2}<0$.
} 
now $\lambda=0$. Rewriting the above yields

$$
\int m\left(w\left(x_{1}\right)\right)\left(\frac{1}{m^{\prime}\left(w\left(x_{1}\right)\right)}-\mu_{2} N^{\prime}\left(a_{2}\right)\right) g^{1}\left(x_{1} \mid a_{1}\right) d x_{1}=\mu_{1} c_{1}\left(a_{1}, a_{2}\right) .
$$

Since $E U_{2}=0$, or $N^{\prime}\left(a_{2}\right)=\frac{c_{2}\left(a_{1}, a_{2}\right)}{M\left(a_{1}\right)}$,

$$
\begin{aligned}
\int \frac{m\left(w\left(x_{1}\right)\right)}{m^{\prime}\left(w\left(x_{1}\right)\right)} g^{1}\left(x_{1} \mid a_{1}\right) d x_{1} & =\mu_{1} c_{1}\left(a_{1}, a_{2}\right)+\mu_{2} \int m\left(w\left(x_{1}\right)\right) \frac{c_{2}\left(a_{1}, a_{2}\right)}{M\left(a_{1}\right)} g^{1}\left(x_{1} \mid a_{1}\right) d x_{1} \\
& =\mu_{1} c_{1}\left(a_{1}, a_{2}\right)+\mu_{2} c_{2}\left(a_{1}, a_{2}\right) .
\end{aligned}
$$

Since $m$ is negative and increasing, the left hand side is negative. In summary,

$$
\mu_{2} c_{2}\left(a_{1}, a_{2}\right)<-\mu_{1} c_{1}\left(a_{1}, a_{2}\right)<0 \text {. }
$$

By the Envelope Theorem, a small increase in $a_{2}$ reduces costs by

$$
\begin{aligned}
\mu_{1} E U_{12}+\mu_{2} E U_{22} & =\mu_{1}\left[M^{\prime}\left(a_{1}\right) N^{\prime}\left(a_{2}\right)-c_{12}\right]+\mu_{2}\left[M\left(a_{1}\right) N^{\prime \prime}\left(a_{2}\right)-c_{22}\right] \\
& =\mu_{1}\left[\frac{c_{1}}{N\left(a_{2}\right)} N^{\prime}\left(a_{2}\right)-c_{12}\right]+\mu_{2}\left[\frac{c_{2}}{N^{\prime}\left(a_{2}\right)} N^{\prime \prime}\left(a_{2}\right)-c_{22}\right] \\
& =\mu_{1} c_{1}\left[\frac{N^{\prime}\left(a_{2}\right)}{N\left(a_{2}\right)}-\frac{c_{12}}{c_{1}}\right]+\mu_{2} c_{2}\left[\frac{N^{\prime \prime}\left(a_{2}\right)}{N^{\prime}\left(a_{2}\right)}-\frac{c_{22}}{c_{2}}\right] \\
& >\mu_{1} c_{1}\left[\frac{N^{\prime}\left(a_{2}\right)}{N\left(a_{2}\right)}-\frac{c_{12}}{c_{1}}\right]-\mu_{1} c_{1}\left[\frac{N^{\prime \prime}\left(a_{2}\right)}{N^{\prime}\left(a_{2}\right)}-\frac{c_{22}}{c_{2}}\right] \\
& =\mu_{1} c_{1}\left(a_{1}, a_{2}\right) \frac{N^{\prime}\left(a_{2}\right)}{N\left(a_{2}\right)} \frac{c_{2}\left(a_{1}, a_{2}\right)}{c_{1}\left(a_{1}, a_{2}\right)} \frac{\partial}{\partial a_{2}}\left(\frac{c_{2}\left(a_{1}, a_{2}\right)}{c_{1}\left(a_{1}, a_{2}\right)} \frac{N\left(a_{2}\right)}{N^{\prime}\left(a_{2}\right)}\right)>0
\end{aligned}
$$

where the second equality uses $E U_{1}=E U_{2}=0$. The first inequality uses the bound on $\mu_{2} c_{2}$ derived earlier, combined with the fact that the term in the last bracket is negative. The second inequality invokes (21). Thus, costs are strictly decreasing on $a_{2} \in\left[\underline{a}_{2}, t\left(a_{1}\right)\right)$. The solution to the stated cost-minimization problem is continuous in $a_{2}$, and hence $a_{2}=t\left(a_{1}\right)$ is the cheapest way of inducing $a_{1}$ on $\left[\underline{a}_{2}, t\left(a_{1}\right)\right]$. Incidentally, the solution to the stated cost-minimization problem may even over-estimate the cost at $a_{2}=t\left(a_{1}\right)$. The reason is that if $t\left(a_{1}\right)=\bar{a}_{2}$, the constraint that $E U_{2}=0$ can be replaced by the weaker $E U_{2} \geq 0$. In other words, implementation costs need not be continuous at $t\left(a_{1}\right)$. 